\title{
Empleo femenino, pobreza y desigualdad
}

\section{Un análisis de microdescomposiciones. Uruguay 1991-2012.*}

\author{
Cecilia Parada** \\ 2014 \\ Tesis de Maestría \\ Maestría en Economía \\ Universidad Nacional de La Plata \\ Director: Leonardo Gasparini
}

\footnotetext{
${ }^{*}$ Agradezco a Leonardo Gasparini por el apoyo a lo largo de este proceso así como por sus valiosos comentarios y sugerencias; a Javier Alejo, Nicolás Badaracco, Noemí Katzkowicz, Cecilia Noboa y Juan Zoloa por los productivos intercambios; a los asistentes del seminario de avances, en particualr a Mariana Marchionni, por sus comentarios; y finalmente a Diego Campoy por su apoyo y reiteradas lecturas de la tesis. Cualquier error que hubiera es de mi entera responsabilidad.

${ }^{* *}$ Centro de Estudios Distributivos, Laborales y Sociales (CEDLAS), Universidad Nacional de La Plata y Consejo Nacional de Investigaciones Científicas y Técnicas (CONICET), Argentina. Contacto: cparada@cedlas.org
} 


\title{
Empleo femenino, pobreza y desigualdad
}

Un análisis de microdescomposiciones. Uruguay 1991-2012.

\section{Resumen}

En este trabajo se estudia el efecto del aumento del empleo femenino sobre la distribución del ingreso en Uruguay entre 1991 y 2012, en particular se analizan los efectos sobre la desigualdad y la pobreza. Se aplica la metodología de microdescomposición propuesta por Bourguignon, Ferreira y Lustig (1998), la cual permite capturar en forma parcial el efecto del aumento de la tasa de empleo femenina. Esta metodología permite distinguir el efecto generado por cambios en la condición de empleo (estar o no empleado) y el producido por modificaciones en las horas trabajadas. Los resultados obtenidos muestran que, a pesar de no ocupar un lugar central cuando se busca explicar la evolución de la distribución del ingreso, el aumento del empleo femenino ha tenido efectos significativos, no solo del punto de vista estadístico. En este sentido, los cambios en el empleo femenino contribuyeron en todo momento a reducir los niveles de pobreza y, si bien tuvo resultados modestos sobre la desconcentración del ingreso al inicio del período, éstos fueron más acentuados hacia el final del mismo. En ambos casos, el efecto horas fue más importante al momento de explicar la caída de los indicadores de distribución que el efecto estado.

\begin{abstract}
This paper analyses the effect of the increase in female employment on income distribution in Uruguay between 1991 and 2012. In particular, this paper studies the impact on inequality and poverty. The methodology employed is a microdecomposition proposed by Bourguignon, Ferreira and Lustig (1998). This methodology allows to partially capture the effect of the increase on female employment and to distinguish between effects generated by changes in employment status (being employed or not) and those produced by changes in worked hours. Results suggest that, the increase in female employment has had statistically significant effects, despite not being the most important when it comes to explaining the evolution of income distribution. Changes in female employment contributed to reducing poverty levels throughout the period studied and, although it had modest results on de concentration of income at the beginning of the period, effects were more pronounced towards the end. In both cases, the effect of changes in worked hours was more important in explaining the fall of distribution indicators than the employment effect itself.
\end{abstract}

Código JEL: C15, C24, D31, J16, J21.

Palabras clave: distribución del ingreso, pobreza, desigualdad, microdescomposiciones, empleo femenino, Uruguay. 


\section{Índice}

1. Introducción 2

2. Literatura previa 4

2.1. Distribución del ingreso en Uruguay y participación de la mujer en el mercado de trabajo . . . . . . . . . . . . . . . . 5 5

2.2. Principales estudios sobre microdescomposiciones . . . . . . . . . . 6

3. Empleo femenino en Uruguay $\quad 7$

4. Estrategia empírica $\quad 13$

5. Estrategia de estimación 14

$\begin{array}{lr}\text { 6. Resultados } & 17\end{array}$

7. Conclusiones 22

8. Referencias bibliográficas 23

A. Apéndice 25 


\section{Introducción}

En las últimas décadas se ha registrado un importante aumento de la tasa de participación femenina en el mercado de trabajo en todo el mundo. En América Latina, durante la segunda mitad del siglo XX se han incorporado al mercado laboral aproximadamente 70 millones de mujeres, lo cual es reflejo de la importante transformación que ha tenido el rol económico de las mujeres en la región (Chioda, 2011).

Las causas detrás del aumento de la cantidad de mujeres ocupadas se encuentran tanto del lado de la demanda, como de la oferta. Factores como el cambio tecnológico, transformaciones en la estructura productiva y el peso de los distintos sectores en la economía, así como mejoras en los sistemas de cuidados, mayor acceso a la educación, avances en la medicina, cambios en la composición de los hogares, son algunos de los motivos que se encuentran detrás del nuevo rol de la mujer (Espino y Leites, 2008).

Por otra parte, si bien la incorporación de las mujeres al mundo del trabajo es un fenómeno que interesa estudiar per se, también interesa estudiarlo por las consecuencias que puede arrojar sobre otros fenómenos como el matrimonio y los divorcios, las tasas de fecundidad, las brechas de ingreso entre hombres y mujeres, la toma de decisiones al interior del hogar (Killingsworth y Heckman, 1986) y, en particular, este trabajo se focaliza en los efectos sobre la distribución del ingreso.

Si consideramos el período comprendido entre 1991 y 2012, en Uruguay se ha producido una importante reducción de la brecha de empleo entre hombre y mujeres. Según datos oficiales, la tasa de empleo masculina, a pesar de haber sufrido fluctuaciones durante el período considerado, en 2012 se ubicó en valores muy cercanos a los de 1991, pasando de $68.2 \%$ a $69.1 \%$. Por su parte, la tasa de empleo femenina mostró una tendencia ascendente durante todo el período, incluso considerando la caída post crisis de 2002, la tasa aumentó de $38.7 \%$ en 1991 a $51.5 \%$ en 2012 (Figura 1). Sin embargo, estos cambios no han sido uniformes, ni durante todo el período, ni a lo largo de la distribución del ingreso. Mientras que entre 1991-2000 la tasa de empleo de las mujeres pertenecientes a hogares del decil más rico aumentó $15.6 \%$, su análoga de los hogares más pobres lo hizo tan solo $3.7 \%$. Por otra parte, entre 2000 y 2012, la situación parece revertirse. La tasa de empleo de las mujeres pertenecientes a los deciles más pobres tuvo un crecimiento algo superior al $28 \%$, mientras que la de los deciles más ricos lo hicieron $11.5 \%$, crecimiento inferior al promedio.

Estos cambios en el mercado de trabajo se dieron en un marco en el cual el PIB per cápita en dólares casi se multiplicó por 2 entre 1991 y 2000, y mostró un incremento aún mayor entre 2000 y 2012. Sin embargo, la pobreza registró valores similares en 1991 y 2000 y la desigualdad (aproximada por el índice de Gini) aumentó 3 puntos. Por otra parte, entre 2000 y 2012, tanto la pobreza como la desigualdad evidenciaron una caída, independientemente del indicador por el cual se las aproxime. Por tal motivo, resulta interesante estudiar de qué forma ha evolucionado la distribución del ingreso y cómo ha impactado sobre esta el aumento de la participación femenina en el mercado de trabajo. Resulta esperable que el incremento de la ocupación femenina tenga un impacto distributivo, tanto sobre la desigualdad como sobre los niveles de pobreza. Un aumento del empleo de las mujeres pertenecientes a los hogares más pobres, dado lo demás constante, es de esperar que produzca una caída de la desigualdad y una reducción de la pobreza. 
Figura 1:

Tasa de empleo. Uruguay 1991-2012.

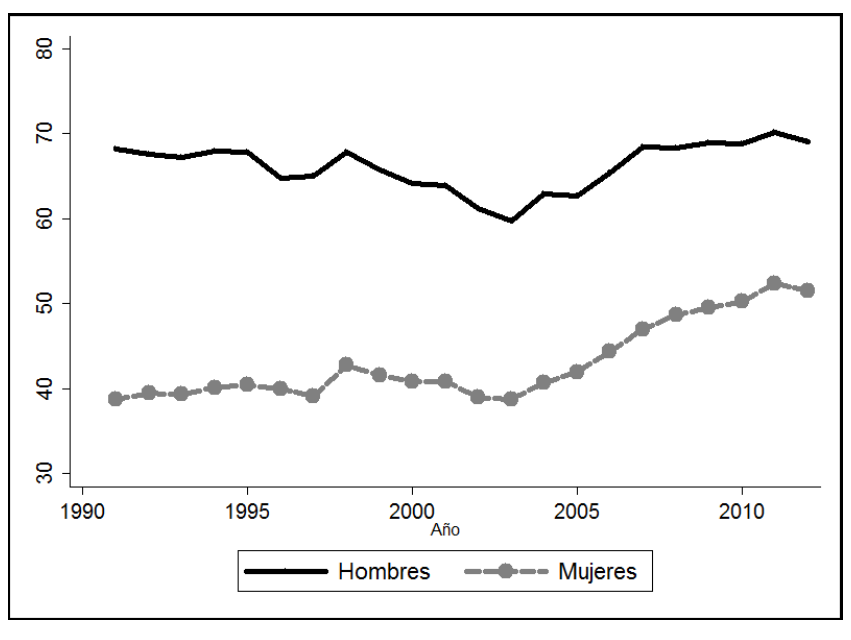

Fuente: INE. Localidades urbanas con más de 5000 habitantes.

Por otra parte, un incremento en el empleo de las mujeres pertenecientes a los quintiles más ricos podría incrementar la desigualdad y no tener efectos sobre los niveles de pobreza.

En este trabajo se intentará cuantificar cuál es la importancia relativa que tuvo el aumento del empleo femenino en las variaciones de la desigualdad y la pobreza. Para ello, se utiliza la metodología de microdescomposición propuesta por Bourguignon, Ferreira y Lustig (1998) y aplicada por Gasparini et al. (2004) para Argentina, la cual permite capturar en forma parcial el efecto del aumento de la tasa de empleo femenina, es decir, los efectos de primer orden. Además del efecto agregado del cambio del empleo femenino, esta metodología permite distinguir entre el efecto generado por cambios en las decisiones de estar empleada (si trabaja o no) de los producidos por modificaciones en las horas trabajadas.

Se supone que los ingresos laborales y las horas trabajadas se determinan simultáneamente en un modelo de equilibrio de mercado laboral, el cual sigue una especificación correspondiente a un Tobit Tipo III según la clasificación de Amemiya (1985). Una vez estimados los parámetros, se simula cuál hubiese sido la distribución de ingresos en el período $t$ si el empleo femenino hubiese sido la del período $t^{\prime}$ y viceversa, bajo el supuesto de que las demás variables se hubiesen mantenido constantes. A partir de allí se estiman indicadores de pobreza y desigualdad y se los compara con los efectivamente observados para cada año. Se considera como el efecto distributivo a la diferencia entre el conjunto de indicadores estimados a partir del contrafáctico y el observado.

Partiendo del hecho de que los cambios en las decisiones de las mujeres de estar ocupadas, o de modificar la cantidad de horas trabajadas, afectan los ingresos de los hogares, el objetivo de este trabajo es estimar el efecto de dichos cambios sobre la distribución del ingreso de Uruguay.

El cambio en las decisiones, posibilidades, de empleo de las mujeres se evalúa sobre 
la pobreza y la desigualdad. En el primero de los casos, se estiman los cambios en el indicador FGT (Foster, Greer y Thorbecke, 1984) con parámetros 0, 1 y 2, es decir, lo que se conoce como incidencia de la pobreza, brecha de la pobreza y profundidad de la pobreza, considerando una línea per cápita de 4 dólares por día a PPA. Para estudiar los efectos sobre la desigualdad se utiliza el índice de Gini sobre el ingreso per cápita familiar. La fuente de información utilizada en este trabajo es el conjunto de microdatos de las Encuestas Continuas de Hogares (ECH) de Uruguay entre 1991 y 2012, publicadas por el Instituto Nacional de Estadística (INE).

Cuando se consideran en forma aislada el efecto estado y el efecto horas, los resultados obtenidos en este trabajo indican efectos relativamente pequeños si se los compara con el efecto empleo total. En particular, aquellos producidos por los cambios el estatus de ocupación son menores a los generados como resultado de cambios en las horas trabajadas. $\mathrm{Al}$ evaluarse el efecto empleo, este es de una magnitud mayor, y explica una parte importante de los cambios en la distribución. Entre 1991 y 2012, los efectos sobre la pobreza son relativamente más importantes que aquellos que ocurren sobre la desigualdad. A pesar de encontrar diferencias en las magnitudes, en los dos sub-períodos analizados se observa que los cambios en el empleo femenino contribuyen a reducir la pobreza y la concentración del ingreso. Estos efectos se encuentran, incluso, cuando la pobreza y la desigualdad aumentan para el conjunto de la población. El efecto empleo total es mayor a la variación del índice de Gini entre 1991 y 2012 y representa el $40 \%$ de la caída experimentada por la tasa de indicendia de la pobreza durante dicho período.

El presente trabajo se organiza de la siguiente manera. En la segunda sección, se realiza una revisión de la literatura teórica y empírica del tema, distinguiendo los antecedentes de tipo metodológicos de aquellos que se han preocupado por la temática para el caso uruguayo. En la tercera sección, se realiza un análisis descriptivo de la evolución del empleo femenino en Uruguay. A continuación, en la cuarta sección, se presenta la estrategia empírica adoptada. Luego, en la quinta sección, se describe la estrategia de estimación realizada y las fuentes de información con las que se trabaja. En la sexta sección, se presentan los resultados obtenidos en forma separada para la pobreza y la desigualdad. Finalmente, en la séptima sección, y a modo de conclusión, se presentan los comentarios finales del estudio.

\section{Literatura previa}

Con el objetivo de tener un marco de referencia para los resultados obtenidos, en este apartado se resumen los principales antecedentes del trabajo. En particular, se distingue entre aquellos estudios que tienen como objetivo el análisis de la distribución del ingreso y de la participación de las mujeres en el mercado de trabajo en Uruguay de los trabajos cuya estrategia empírica consistió en la realización de microdescomposiciones basadas en la metodología propuesta por Bourguignon, Ferreira y Lustig (1998). 


\subsection{Distribución del ingreso en Uruguay y participación de la mujer en el mercado de trabajo}

Dentro de los trabajos que analizan la distribución del ingreso en Uruguay durante los últimos años se destacan los realizados por Marroig y Oreiro (2008) y el de Alves, Amarante, Salas y Vigorito (2012). El primero de estos trabajos se propone analizar la evolución de la desigualdad y la pobreza en Uruguay entre 1991 y 2005, para lo cual aplican una metodología de microsimulaciones que permite descomponer el cambio total en la distribución entre distintos efectos. Las autoras concluyen que los retornos a la educación, los años de estudio y las jubilaciones son los elementos que explican en mayor medida la distribución del ingreso. De esta manera, encuentran que los retornos a la educación tienen un efecto desigualador y empobrecedor, mientras que el aumento en los años de estudio contribuye a explicar la disminución de la pobreza y las jubilaciones condicionan, de cierta forma, el perfil etario de la pobreza, recayendo en los grupos más jóvenes.

Por su parte, Alves et al. (2012) analizan la evolución y principales determinantes de la desigualdad de ingresos en Uruguay durante el período comprendido entre 1986 y 2009. En base a un análisis de descomposición del ingreso por fuentes, los autores encuentran que los ingresos laborales y la instauración de sistemas no contributivos de transferencia fueron los principales factores al momento de explicar la evolución de la desigualdad. Con el fin de identificar determinantes de la desigualdad de ingresos laborales realizaron microsimulaciones. Al igual que en el trabajo precedente, encontraron que los retornos a la educación son los que explican en mayor medida los cambios en la desigualdad. Por su parte, el crecimiento del empleo en los últimos años de su análisis, junto con la reducción de los diferenciales regionales, tuvieron un efecto igualador, mientras que la cantidad de años de estudio contribuyeron muy poco a explicar los cambios en la desigualdad.

En lo que respecta al rol de la mujer en el mercado de trabajo y su incidencia en la distribución del ingreso, se destacan sobre el resto tres trabajos que se ocupan de la temática para Uruguay. Por un lado, el trabajo de Gónzalez y Rossi (2003) "Participacion femenina en el mercado de trabajo: efectos sobre la distribucion del ingreso en Uruguay", por otro lado, el trabajo de Espino (2003) "El aporte de las remuneraciones femeninas en los hogares y sus efectos en la distribucion del ingreso" y, finalmente, el de Vigorito (1999) "Una distribución del ingreso estable. El caso de Uruguay 1986-1997".

En la investigación llevada adelante por Gónzalez y Rossi (2003), los autores analizan el impacto de la mayor participación femenina en el mercado de trabajo uruguayo sobre la equidad para el período comprendido entre 1986 y 1997. En dicho estudio concluyen que todas las fuentes contribuyen en forma positiva a la desigualdad total entre hogares, lo cual se debe a la correlación positiva existente entre todas ellas y el ingreso del hogar.

Por su parte, Espino (2003) analiza los efectos sobre el bienestar de la incorporación de las mujeres al mercado laboral entre 1987 y 2000. Cuantifica la participación económica de las mujeres y de los ingresos laborales que generan, así como su aporte a los cambios en la distribución del ingreso global. A partir de la descomposición de los índices de desigualdad la autora concluye que, los ingresos laborales de las mujeres contribuyen positivamente a la concentración del ingreso de los hogares, a pesar de que la misma es menor a la contribución realizada por los ingresos masculinos. 
Asimismo, Vigorito (1999) analiza en qué medida la incorporación al mercado de trabajo de las mujeres, así como de otros miembros del hogar, influyó sobre la desigualdad de ingresos de los hogares urbanos. Como resultado encuentra que, entre 1986 y 1997 se redujo la contribución de los hombres jefes de hogar a la concentración del ingreso, mientras que aumento la participación de jefas y cónyuges. Al tiempo que las mujeres incrementaron su contribución a la generación de ingresos de los hogares, aumentaron su contribución a la desigualdad en mayor medida, debido a que todas sus fuentes tienen una mayor dispersión que la de los hombres.

\subsection{Principales estudios sobre microdescomposiciones}

Del punto de vista metodológico, las microsimulaciones aplicadas al análisis distributivo permiten descomponer las fuentes del cambio en los ingresos de los hogares y los efectos sobre las medidas de desigualdad y pobreza. Los primeros trabajos que utilizaron esta metodología fueron llevados adelante por Blinder (1973) y Oaxaca (1973), quienes la emplearon con el fin de estudiar las diferencias en las medias de dos distribuciones. Más adelante Juhn, Murphy y Pierce (1993) ampliaron este enfoque logrando considerar diferencias a lo largo de toda la distribución, superando la limitación de comprar los comportamientos promedios. Posteriormente, Bourguignon, Ferreira y Lustig (1998) generalizaron el enfoque, permitiendo que sea empleado sobre diversas formas funcionales. Este último enfoque fue adaptado para Argentina por Gasparini, Marchionni y Sosa Escudero (2000), y posteriormente retomado por un conjunto de trabajos, algunos de los cuales se destacan a continuación.

En el trabajo realizado en el marco del convenio entre el Ministerio de Economía de la Provincia de Buenos Aires y la Facultad de Ciencias Económicas de la Universidad Nacional de La Plata, Gasparini et al. (2000) estudian los determinantes de la desigualdad en la distribución del ingreso desde distintas aproximaciones. A los efectos de este estudio, interesa destacar su trabajo sobre el empleo y la desigualdad desde un análisis de microsimulaciones realizado para el período 1986-1998. Al igual que en esta investigación, ponen el énfasis en analizar los efectos sobre la desigualdad ocasionados por los cambios en el empleo y la cantidad de horas trabajadas. Encuentran que los cambios en la tasa de empleo, si bien tuvieron un efecto desigualador, fueron muy pequeños. Por su parte, los cambios en las horas trabajadas parecen haber tenido un efecto relevante.

A partir del trabajo anterior, Gasparini, Marchioni y Sosa Escudero (2004) evalúan nuevamente los factores que afectaron la desigualdad en el Gran Buenos Aires en el período comprendido entre 1986 y 1998. En su trabajo distinguen dos sub-períodos: 1986-1992 y 1992-1998. Mientras que durante el primer sub-período encuentran que el modesto cambio ocurrido en la desigualdad se debió a fuerzas leves que se compensaron entre sí, entre 1992 y 1998, hallan que prácticamente todos los factores tuvieron un papel en el aumento de la desigualdad. De esta forma, para el segundo sub-período, observan que los retornos a la educación, los cambios en la dotación de factores no observables, así como sus remuneraciones, y la caída de las horas de trabajo en las personas de bajos ingresos, contribuyeron a incrementar la desigualdad. Lo anterior, sumado a una baja incidencia redistributiva de la disminución de la brecha salarial de géneros, la tasa de desempleo y la estructura 
educativa, condujeron en Argentina a un aumento de la desigualdad sin precedentes.

En la misma línea que los trabajos citados, pero analizando un período de tiempo más cercano, Zoloa (2008) analiza los cambios en la distribución del ingreso producidos en Argentina entre 1998 y 2005. Si bien la desigualdad de ingresos se mantuvo estable en el período considerado, el autor encuentra importantes efectos distributivos de algunos factores. En particular, los retornos a la educación tuvieron un efecto desigualador importante, mientras que el aumento de los factores inobservables junto con los mayores niveles de empleo tuvieron el efecto contrario.

\section{Empleo femenino en Uruguay}

En Uruguay en las últimas décadas, tanto la participación de la mujer en el mercado de trabajo como la distribución del ingreso y la pobreza han sufrido variaciones importantes. Sin pretender establecer una relación causal, en este apartado se presenta evidencia sobre los fenómenos a analizar a lo largo del trabajo. El análisis se concentra en el periodo 1991-2012, con especial énfasis en los años 1991, 2000 y 2012, para los cuales se presenta información resumida sobre la evolución de la distribución del ingreso y el empleo femenino. Con el fin de analizar la evolución de la pobreza y la desigualdad, se estima la tasa de incidencia de la pobreza, considerando una Línea de Pobreza moderada de 4 dólares por día por individuo, y el índice de Gini sobre el ingreso per cápita familiar (IPCF). Por su parte, a los efectos de la construcción de la tasa de empleo femenina (TEF), se considera al total de mujeres de entre 14 y 64 años de edad, quienes constituyen el grueso de la fuerza laboral de las mujeres.

Como se adelantó en la Introducción, la fuente de información utilizada a lo largo de todo este trabajo es el conjunto de microdatos de las Encuestas Continuas de Hogares (ECH) de Uruguay entre 1991 y 2012, las cuales son publicadas por el Instituto Nacional de Estadística (INE). Las ECH contienen información de personas y hogares para todo el territorio nacional. Debido a que la representatividad de la ECH de Uruguay ha ido cambiando, a los efectos de que las estimaciones realizadas sean comparables par todos los años, se trabaja con datos representativos de localidades urbanas con más de 5,000 habitantes.

Entre 1991 y 2012, la distribuciónd el ingreso en Uruguay sufrió importantes variaciones. En un principio, durante la década del noventa, al igual que lo registrado en el resto del continente latinoamericano, la pobreza y la desigualdad mostraron un fuerte incremento. Sin embargo, durante la primera década del siglo XXI, en particular luego de 2003, la tendencia ascendente de estos indicadores comenzó a revertirse.

En las Figuras 2 y 3 se ilustra la evolución de dos dimensiones de la distribución del ingreso en forma conjunta con la tasa de empleo de las mujeres. Las oscilaciones de los indicadores han mostrado diferentes tendencias entre 1991 y 2012, pudiendo diferenciar dos sub-periodos. En lo que respecta a la evolución de la pobreza se observa que, luego de una caída en 1992, comienza a incrementarse lentamente hasta que a finales de la década del noventa sufre un drástico aumento. Esto condujo a que en 2004 cerca del $25 \%$ de la población se encontrase por debajo de la línea de pobreza. Sin embargo, luego del pico 
Figura 2:

Evolución de la pobreza. Uruguay

1991-2012.

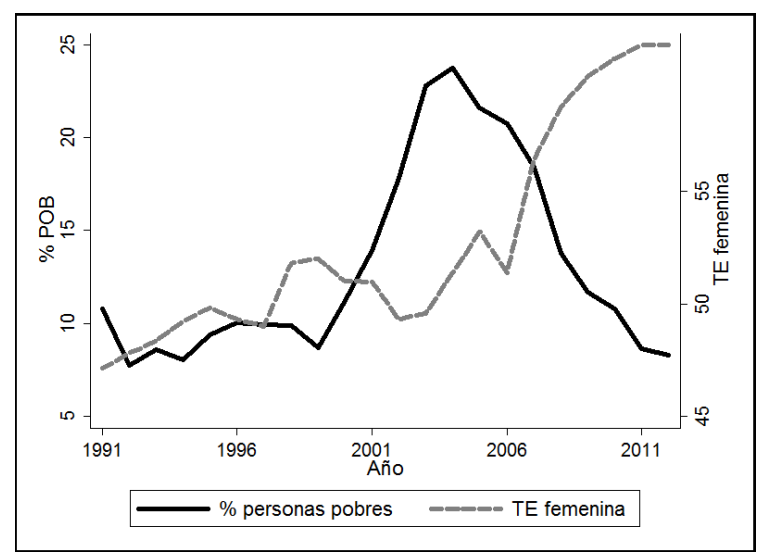

Fuente: Elaboración propia en base a ECH. Nota: Localidades urbanas con más de 5000 habitantes.
Figura 3:

Evolución de la desigualdad. Uruguay 1991-2012.

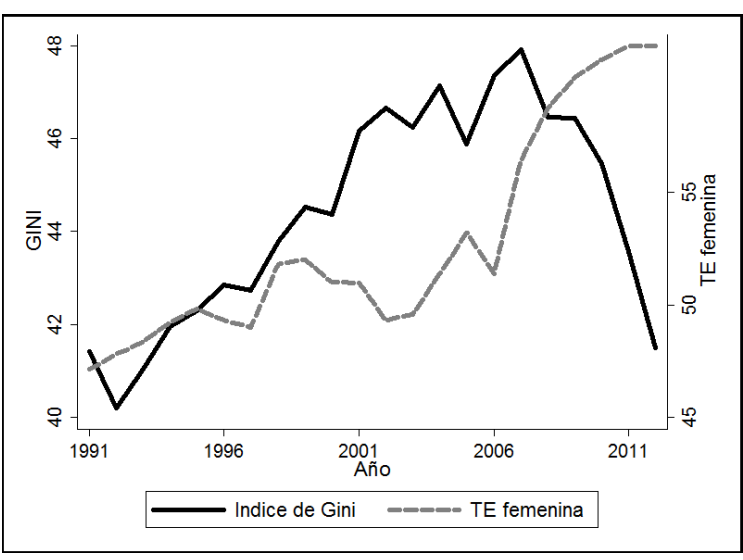

Fuente: Elaboración propia en base a ECH. Nota: Localidades urbanas con más de 5000 habitantes.

registrado en dicho año, la tendencia de la tasa de incidencia de la pobreza se revierte, ubicandose en 2012 en $8.2 \%$, el valor más bajo de los últimos 20 años.

Por su parte, la desigualdad, aproximada por el índice de Gini, ha mostrado una evolución similar a la de la pobreza pero con sus particularidades. A partir del año 1992 se inició una etapa de fuerte crecimiento de la concentración del ingreso, que alcanzó sus valores máximos en 2007. La desigualdad, a diferencia de la pobreza que aumentó especilamente a finales de la década del noventa, mostró una tasa de crecimiento casi constante durante toda la década y, recién en 2007, tres años más tarde que la pobreza, comenzó a registrar variaciones negativas.

Tanto en la figura 2 como en la 3, se muestra la evolución de la tasa de empleo femenina con el fin de dar una referencia al lector. A diferencia de los indicadores de pobreza y desigualdad, la TEF evidenció un aumento durante todo el período, con excpeción del año 2002 como reflejo de la importante crisis que atravesaba el país. En particular, entre 2006 y 2012, la tasa de empleo de las mujeres aumentó 10 puntos porcentuales, alcanzando el $61.5 \%$ para la población de referencia. ${ }^{1}$

La evolución de la tasa de empleo femenina no ha sido homogénea si se agrupa a la población de acuerdo distintas variables. Distinguiendo entre dos sub-períodos: 1991-2000 y 2000-2012, en las Figuras 4a y 4b puede observarse la variación de la TEF según el decil de ingresos del hogar al que pertenecen las mujeres. La línea horizontal es indicativa de la variación de la TEF promedio. A partir de dichas figuras puede apreciarse que, entre 1991 y 2000, las mujeres pertenecientes a hogares del primer decil de ingresos tuvieron un aumento de su tasa de empleo de $4.0 \%$, registrando una variación menor al promedio $(8.2 \%)$, mientras que las mujeres pertenecientes a los hogares más ricos, fueron, junto con las del séptimo decil, quienes registraron el mayor crecimiento de su tasa de empleo,

\footnotetext{
${ }^{1}$ La tasa de empleo femenina que se observa es mayor a la presentada en la Figura 1. La diferencia se debe a que en esta oportunidad se considera al universo de mujeres de entre 14 y 64 años de edad, mientras que en el caso anterior se consideraban a todas las mujeres mayores de 13 años.
} 
$16.2 \%, 8$ puntos porcentuales superior al promedio. Este escenario se revierte en el subperíodo siguiente. Entre 2000 y 2012, las mujeres que mostraron un mayor crecimiento de su tasa de ocupación fueron aquellas pertenecientes a hogares de los dos deciles más pobres (28\% aproximadamente), mientras que las pertenecientes al decil más rico, si bien aumentaron su nivel de ocupación (11.5\%), lo hicieron por debajo del promedio general (20.6\%).

Figura 4:

Variación de la TEF según decil de ingreso del hogar: 1991-2012.

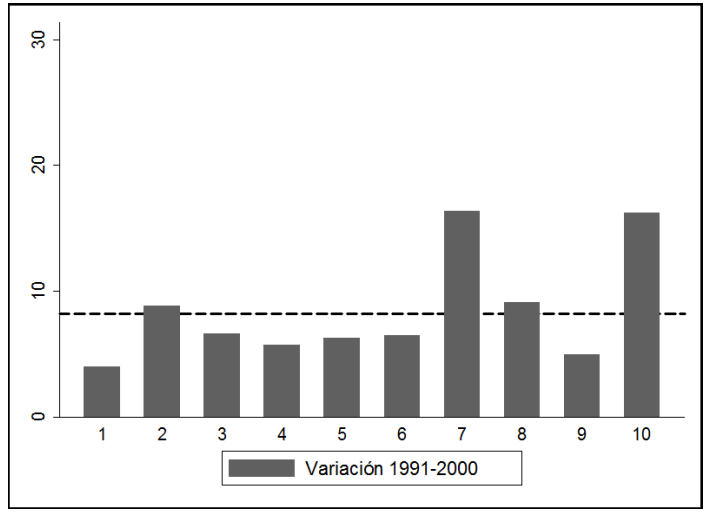

(a) Período 1991-2000

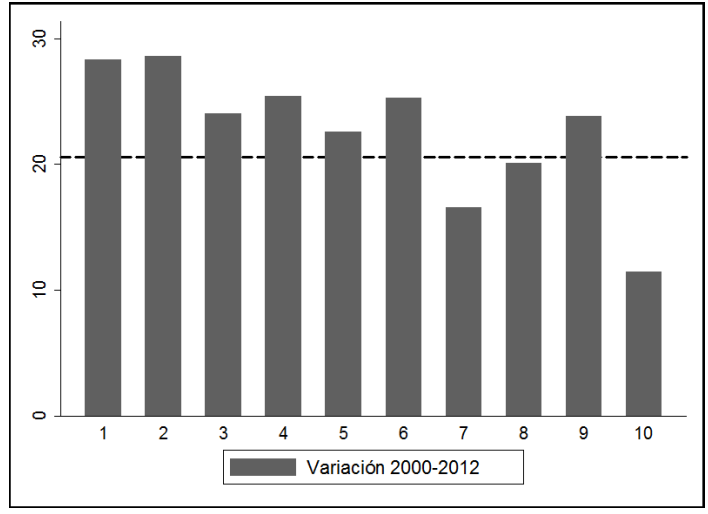

(b) Periodo 2000-2012.

Fuente: Elaboración propia en base a ECH.

Nota: Localidades urbanas con más de 5000 habitantes.

Además de la variable dicotómica, de estar empleada o no, resulta pertinente analizar la evolución de las horas trabajas. En la Tabla 1, se presenta por decil de ingresos del hogar las horas semanales trabajadas promedio por las mujeres en 1991, 2000 y 2012. Se observa que, entre 1991 y 2000 las trabajadoras de los hogares del décimo decil mostraron una cantidad de horas trabajadas relativamente estable, mientras que las mujeres pertenecientes a los hogares más pobres, sufrieron una variación negativa de las horas promedio trabajadas. Por otra parte, durante la primera década del siglo XXI las mujeres pertenecientes a todos los grupos de ingresos registraron una caída en la cantidad de horas trabajadas promedio, siendo la más importante la registrada por las mujeres más pobres. Es decir, el aumento de a la tasa de empleo observado en la Figura 4, se dio en un marco de disminución de las horas promedio.

En la Tabla 2 se desagrega la información sobre la situación de empleo de las mujeres de acuerdo al grupo etario al que pertenecen. A tales efectos se distinguen cuatro grupos: mujeres de entre 14 y 24 años de edad, de entre 25 y 40, de entre 41 y 64 y de 65 y más. El segundo grupo es el que muestra en todos los períodos una mayor tasa de empleo, seguido por el tercer grupo, mientras que las menores tasas las registran las mayores de 65 años. Al mismo tiempo, al considerar las variaciones de las tasas específicas, se puede observar que la TEF aumentó en mayor medida para los grupos más envejecidos. De hecho, el único caso en que se registró una leve variación negativa fue para las mujeres de entre 14 y 24 años entre 1991 y 2000.

Otra forma de distinguir grupos dentro del total de mujeres es de acuerdo al máximo nivel educativo alcanzado. De este modo, en la Tabla 3 se muestra la TEF según nivel 
Tabla 1:

Horas promedio trabajas por decil de ingresos.

\begin{tabular}{cccc}
\hline \hline Decil & 1991 & 2000 & 2012 \\
\hline 1 & 33.9 & 32.1 & 30.0 \\
2 & 36.9 & 35.4 & 33.8 \\
3 & 38.1 & 37.1 & 36.3 \\
4 & 39.5 & 38.1 & 36.6 \\
5 & 39.8 & 37.8 & 37.6 \\
6 & 39.9 & 38.8 & 37.9 \\
7 & 41.1 & 39.7 & 38.2 \\
8 & 40.5 & 40.8 & 38.7 \\
9 & 39.8 & 39.4 & 39.2 \\
10 & 42.0 & 42.4 & 41.1 \\
\hline Total & 39.4 & 38.2 & 36.9 \\
\hline \hline
\end{tabular}

Fuente: Elaboración propia en base a ECH. Nota: Localidades urbanas con más de 5000 hab.

Tabla 2:

Tasa de ocupación de acuerdo al grupo etario.

\begin{tabular}{crrr}
\hline \hline Grupo de edad & 1991 & 2000 & 2012 \\
\hline$[14,24]$ & 30.8 & 30.2 & 31.4 \\
{$[25,40]$} & 62.2 & 65.2 & 76.4 \\
{$[41,64]$} & 46.2 & 53.9 & 68.0 \\
{$[65+]$} & 5.6 & 6.3 & 10.3 \\
\hline Total & 39.0 & 41.4 & 50.5 \\
\hline \hline
\end{tabular}

Fuente: Elaboración propia en base a ECH

Nota: Localidades urbanas con más de 5000 hab.

educativo para las mujeres de entre 14 y 64 años de edad, que conforman el grueso de la fuerza laboral femenina. En primer lugar, se destaca la relación positiva entre nivel educativo y tasa de empleo, la cual es persistente a lo largo del tiempo. Por otra parte, puede observarse, que la tasa de empleo no varió en forma homogénea si se distingue por grupo educativo. Las mujeres con educación superior o universitaria completa fueron las que registraron en todos los casos la mayor tasa de empleo y, al mismo tiempo, fueron quienes mostraron el menor crecimiento relativo en la ocupación entre 1991 y 2012. En el otro extremo, las mujeres con primaria incompleta, registraron en todos los años la menor tasa de ocupación. Sin embargo, mientras entre 1991 y 2000 tuvieron una leve caída de su tasa de empleo, entre 2000 y 2012, aumentaron su ocupación un $10 \%$. Si se considera todo el período analizado, las mujeres con primaria y secundaria completa fueron quienes mostraron el mayor crecimiento de su tasa de empleo, de aproximadamente $28 \%$.

Finalmente, en la Tabla 4 se distinguen grupos de mujeres de acuerdo al rol que ocupan dentro del hogar, es decir: jefas, cónyuges u otras. Si bien las jefas tienen una tasa de empleo muy por encima del resto de los grupos, el mayor crecimiento se evidenció en las cónyuges, quienes pasaron de registrar guarismos por debajo del promedio en 1991 a 
Tabla 3:

Tasa de ocupación según nivel educativo.

\begin{tabular}{lrrr}
\hline \hline Nivel educativo & 1991 & 2000 & 2012 \\
\hline Primaria incompleta & 34.9 & 34.7 & 38.3 \\
Primaria completa & 43.0 & 43.3 & 55.0 \\
Secundaria incompleta & 44.4 & 46.1 & 53.8 \\
Secundaria completa & 59.8 & 70.6 & 76.9 \\
Superior incompleta & 52.1 & 54.0 & 63.0 \\
Superior completa & 84.6 & 85.8 & 89.0 \\
\hline Total & 47.1 & 51.0 & 61.5 \\
\hline \hline
\end{tabular}

Fuente: Elaboración propia en base a ECH.

Nota: Localidades urbanas con más de 5000 hab.

superarlo en 2012. Por su parte, las mujeres que integran el resto de los integrantes del hogar (otras), han mostrado una tasa de empleo bastante estable, en el entorno del $42 \%$.

Tabla 4:

Tasa promedio de ocupación según posición en el hogar.

\begin{tabular}{lrrr}
\hline \hline Posición en el hogar & 1991 & 2000 & 2012 \\
\hline Jefe & 62.3 & 64.6 & 77.3 \\
Cónyuge & 45.1 & 51.5 & 64.8 \\
Otras & 41.3 & 42.3 & 42.4 \\
\hline Total & 47.1 & 51.0 & 61.5 \\
\hline \hline
\end{tabular}

Fuente: Elaboración propia en base a ECH. Nota: Localidades urbanas con más de 5000 hab.

Si bien la relación no es unidireccional, es de esperar que los cambios en el comportamiento de las mujeres respecto al empleo tengan consecuencias sobre la estructura de los ingresos en los hogares. En particular, si se descompone el ingreso del hogar por fuentes puede distinguirse ingreso laboral femenino, ingreso laboral masculino y otros ingresos. $\mathrm{Al}$ observar la evolución de las distintas fuentes se pueden distinguir algunas tendencias. Por un lado, que el ingreso laboral femenino tiene un menor peso en los hogares pertenecientes a los deciles extremos, lo cual se ha acentuado en el último período. Por otro lado, entre 1991 y 2012, se observa un aumento en la participación del ingreso laboral femenino en el ingreso total del hogar, independientemente del decil de ingresos que se considere. Estos elementos tienen como contrapartida, una disminución del peso del ingreso laboral masculino en el ingreso total para todos los deciles entre 1991 y 2012, junto con un aumento del peso de otros ingresos en los hogares de deciles extremos y una caída de su participación en los hogares ubicados entre el segundo y séptimo decil.

Si bien, a partir del análisis descriptivo anterior no puede concluirse acerca de la relación entre la evolución del empleo femenino y la distribución del ingreso, es posible plantear algunas hipótesis que guíen este trabajo. Siguiendo a Gasparini et al. (2000), el aumento de la cantidad de mujeres empleadas tendría efectos sobre la pobreza y el 
Figura 5:

Participación de las distintas fuentes de ingreso según decil: 1991-2000-2012.

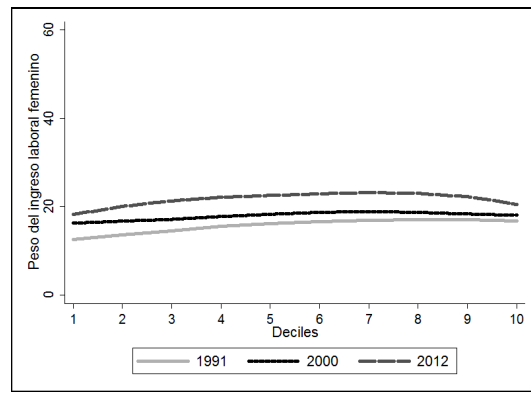

(a) Ingreso laboral femenino.

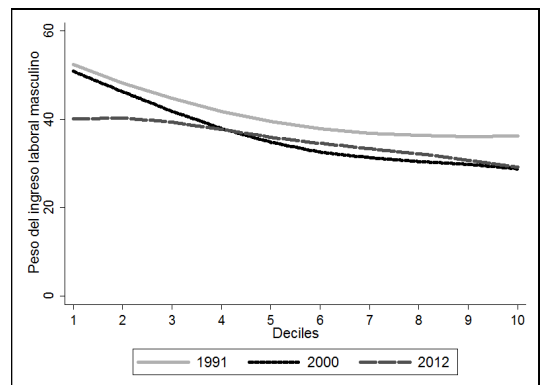

(b) Ingreso laboral masculino.

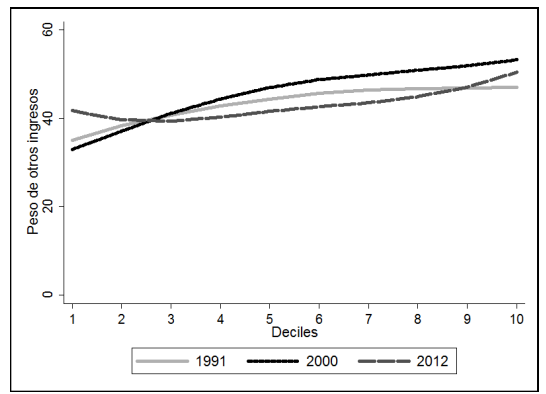

(c) Otros ingresos del hogar.

Fuente: Elaboración propia en base a ECH.

Nota: Localidades urbanas con más de 5000 habitantes.

desempleo, al menos a través de dos canales: por un lado, debido a que un mayor número de personas tiene ingresos laborales positivos, es menor la cantidad de individuos con ingreso cero. Ello produciría una reducción de la cantidad de personas pobres y una caída en la desigualdad. Por otro lado, dependiendo del grupo socioeconómico al que pertenecen las mujeres que consiguen empleo, podría producirse que la desigualdad aumente a pesar de que la pobreza disminuya o se mantenga estable. De todas formas, podría esperarse que, a medida que más mujeres se van incorporando al mercado laboral, y aumenta la demanda de éstas por parte de los empleadores, aumenta la probabilidad de que quienes estén menos educadas consigan empleo y, por lo tanto, se produzca una reducción de la desigualdad. En este trabajo se procura estudiar la importancia de estos canales para el caso uruguayo entre 1991 y 2012. 


\section{Estrategia empírica}

En este apartado se detalla la metodología de descomposiciones empleada a los efectos de evaluar el impacto sobre la distribución del ingreso de los cambios producidos en las decisiones de empleo de las mujeres, distinguiendo los cambios en las horas trabajadas de las decisiones de estatus ocupacional de las mujeres. Es decir, bajo el supuesto de que todo lo demás se mantiene constante, la distribución del ingreso puede cambiar como resultado tanto de las decisiones, o posibilidades, de estar ocupadas y/o por cambios en la cantidad de horas trabajadas que realizan las mujeres. Concretamente, se emplea la metodología propuesta por Bourguignon, Ferreira y Lustig (1998) y aplicada por Gasparini at al. (2004) para al caso argentino. Esta metodología permite aproximarse a la medida en que los cambios en las decisiones laborales de las mujeres contribuyen a explicar las diferencias en la distribución del ingreso y la pobreza en los distintos momentos del tiempo.

Sin embargo, este método presenta algunas limitaciones que se deben tener presente al momento de interpretar los resultados. En particular, las microdescomposiciones consideran que la estructura condicional de la población se mantiene inalterada, es decir, supone que no se producen efectos de equilibrio general. En este sentido, se aíslan comportamientos que son endógenos y se consideran independientes. Por ello, se dice que esta metodología permite una aproximación parcial al fenómeno que se quiere estudiar ${ }^{2}$.

A continuación, se describe la metodología empleada. En primer lugar, el ingreso total de los hogares, es aquel compuesto por los ingresos laborales $(L)$ y los no laborales $(N L)$ de todos los individuos $i$ que componen el hogar $h$.

$$
Y_{h t}=\sum_{i=1}^{N}\left(Y_{i t}^{L}+Y_{i t}^{N L}\right)
$$

Donde los ingresos no laborales se consideran determinados exógenamente y, los ingresos laborales de un momento del tiempo $t$ son el resultado del producto entre el ingreso laboral horario $w$ y las horas trabajadas $L$.

$$
Y_{i t}^{L}=w_{i t} . L_{i t}
$$

$w$ y $L$ dependen de características observables, $X_{i t}$, e inobservables, $\varepsilon_{i t}$, así como de los vectores de parámetros $\beta_{t}$ y $\lambda_{t}$, que representan los coeficientes que determinan la forma en que las características observables afectan el salario horario y el número de horas trabajadas, respectivamente.

$$
Y_{i t}^{L}=F\left(X_{i t}, \varepsilon_{i t}, \beta_{t}, \lambda_{t}\right)
$$

A los efectos de este trabajo, se realiza el supuesto de que los ingresos no laborales no se modifican como resultado de cambios en el ingreso laboral.

Dado que el bienestar de un individuo no depende únicamente de su ingreso, se considera como variable de análisis el ingreso per cápita del hogar, el cual se define como,

\footnotetext{
${ }^{2}$ Un análisis más detallado de las limitaciones de la metodología de microdescomposiciones puede encontrarse en Fortin, N., Lemieux, T. y Firpo, S. (2011).
} 


$$
y_{i t}=\frac{\sum_{i=1}^{N}\left(F\left(X_{i t}, \varepsilon_{i t}, \beta_{t}, \lambda_{t}\right)+Y_{i t}^{N L}\right)}{N_{h}} \quad \forall i \epsilon h
$$

Si se considera $k$ un parámetro cualquiera de los argumentos de la función del ingreso laboral (4), luego puede definirse a $Y_{i t}^{L}\left(k_{t^{\prime}}\right)$ como el ingreso que obtendría el individuo $i$ en el momento $t$ si solamente el parámetro $k$ tomara el valor correspondiente al momento $t^{\prime}$, ceteris paribus, obteniendo de esta forma un nuevo ingreso simulado.

De este modo, puede obtenerse una distribución del ingreso per cápita familiar observada y una simulada: $D_{t}$ y $D_{t k}$, respectivamente:

$$
\begin{gathered}
D_{t}=\left\{y_{1 t}, \ldots, y_{N t}\right\} \\
D_{t k}=\left\{y_{1 t}\left(k_{t^{\prime}}\right), \ldots, y_{N t}\left(k_{t^{\prime}}\right)\right\}
\end{gathered}
$$

Luego, con el fin de observar los efectos sobre la dsigualdad de la distribución del ingreso y la pobreza, se calcula el impacto del cambio en el parámetro $k$ sobre un conjunto de indicadores distributivos $I\left(D_{t}\right)$ :

$$
I\left(D_{t k}\right)-I\left(D_{t}\right)
$$

La diferencia entre el valor del indicador de la distribución observada y la simulada se interpreta como la contribución del cambio del parámetro $k$ entre el momento $t$ y $t^{\prime}$ al cambio en la medida distributiva que se estudie.

\section{Estrategia de estimación}

En este trabajo se procurará estimar el efecto del cambio en los parámetros que relacionan las características de los individuos (las mujeres) con la cantidad de horas trabajadas por estos $\left(\lambda_{t}\right)$. Se distinguirá entre el impacto que genera el cambio en la cantidad de horas trabajadas, del efecto provocado por el cambio de status laboral de los individuos. Adicionalmente, se estimarán los efectos producidos por cambios en los precios, $\left(\beta_{t}\right)$, es decir, por cambios en los retornos a la educación y la experiencia de las mujeres en el mercado laboral. Para ello se mide el impacto que producen cambios en los parámetros que relacionan el nivel educativo y la experiencia con el salario horario, respectivamente. De la misma forma, se mide el efecto de cambios en los factores inobservables de las mujeres y sus remuneraciones que afectan el salario horario $\left(\varepsilon^{w}\right)$.

Por lo tanto, para poder estimar el efecto sobre la pobreza y la desigualdad producido por los cambios en los parámetros que relacionan las características de las mujeres con su salario y con la cantidad de horas trabajadas, se deberán realizar estimaciones, tanto de los parámetros del modelo descrito como del término de error.

El modelo cuenta con dos ecuaciones que se describen a continuación; una correspondiente a los salarios horarios $(w)$ y otra al número de horas trabajadas $(L)$.

$$
w_{i t}^{*}=X_{i t}^{w} \beta_{t}+\varepsilon_{i t}^{w}
$$




$$
L_{i t}^{*}=X_{i t}^{L} \lambda_{t}+\varepsilon_{i t}^{L}
$$

$$
\begin{array}{lcc}
\text { Con: }\left(\varepsilon_{i t}^{w}, \varepsilon_{i t}^{L}\right) \sim N\left(0,0, \sigma_{w}, \sigma_{L}, \rho\right) \\
w_{i t}=w_{i t}^{*} \quad \text { si } & L_{i t}^{*}>0 \\
w_{i t}=0 & \text { si } & L_{i t}^{*} \leq 0 \\
L_{i t}=L_{i t}^{*} & \text { si } & L_{i t}^{*}>0 \\
L_{i t}=0 & \text { si } & L_{i t}^{*} \leq 0
\end{array}
$$

Donde $w_{i t}$ representa los valores observados del individuo $i$ en el momento $t$ del salario horario, y $L_{i t}$ su homólogo de las horas trabajadas, mientras que $X_{i t}^{w}$ y $X_{i t}^{L}$ son los vectores de características observables que afectan al salario y las horas trabajadas del individuo $i$ en el momento $t$, respectivamente. De esta forma, $w^{*}$ y $L^{*}$ son variables latentes; solo se observarán valores si el individuo efectivamente trabaja.

La estimación de la ecuación de salarios horarios se realiza mediante el método de selección de Heckman por máxima verosimilitud, coincidiendo la ecuación de selección con la de horas semanales trabajadas, con la diferencia de que se sustituye la variable dependiente de horas trabajadas por una variable dummy que adopta el valor 1 cuando el individuo trabaja y 0 en caso contrario. Por su parte, la ecuación de horas trabajadas se estima mediante un modelo Tobit estándar para datos censurados.

Mediante el procedimiento descrito, se podrán obtener los salarios estimados para los individuos que trabajan, sin embargo, resta determinar los salarios que tendrían aquellas mujeres que no trabajan. La principal dificultad radica en que no se observa el salario de estas personas y, por lo tanto, no es posible obtener el residuo de las ecuaciones (8) y (9). Para resolverlo, se emplean los parámetros estimados de $\hat{\sigma_{w}}, \hat{\sigma_{L}} \mathrm{y} \hat{\rho} \mathrm{y}$ se generan aleatoriamente pares de errores a partir de un muestreo de una distribución normal bivariada, la cual está implícita en el modelo de Heckman. En caso de que los residuos así generados no resulten consistentes con la decisión original de no trabajar, se realiza nuevamente el muestreo de errores para ese individuo, hasta que sea coherente con su comportamiento observado. Luego, se estiman los salarios que tendrían estas mujeres en caso de trabajar, utilizando los errores generados y los coeficientes de $\hat{\beta}_{t}$ estimados.

Los efectos sobre la distribución del ingreso debido a los cambios producidos en la tasa de empleo femenina (EfectoEmpleo) pueden separarse en dos. Por un lado, el impacto ocasionado por el aumento de las horas trabajadas de aquellas mujeres que se encontraban ocupadas (EfectoHoras) y, por otro lado, la incorporación de más mujeres al mercado laboral (EfectoEstado).

El efecto empleo agregado se obtiene simulando el ingreso laboral que obtendrían las mujeres en el período $t^{\prime}$ si las decisiones sobre la cantidad de horas trabajadas y de condición de ocupación respondieran a los parámetros del período $t$. 
Con el fin de estimar el efecto horas, se simula la cantidad de horas trabajadas empleando los parámetros de las ecuaciones estimadas para el período $t^{\prime}$, sin considerar a las personas que cambian su condición de ocupación. Es decir, solo se consideran los cambios en las horas trabajadas de aquellas mujeres que efectivamente trabajan en el año base.

Por otra parte, el efecto estado, simula la distribución del ingreso que hubiese existido en el período $t^{\prime}$ considerando solamente los cambios en las decisiones binarias de empleo de las mujeres. Se simulan las decisiones de empleo considerando los parámetros estimados para el período $t^{\prime}$. Luego, para aquellas mujeres que resultan trabajar en la simulación se les imputa la misma cantidad de horas trabajadas que en el período $t$, en caso de que lo hicieran. Si no se encontraban trabajando en el período $t$ se les imputan horas simuladas con los parámetros del período $t$. Para quienes no resulten trabajar en la simulación se les imputa ingresos cero.

Para estimar los retornos a la educación y la experiencia, se simula la distribución del ingreso que hubiese existido en el período $t^{\prime}$ si los parámetros $\beta^{e d u}$ y $\beta^{e x p}$, que relacionan las características de las mujeres con su salario horario, fuesen los del período $t$. Por lo tanto, se cambian los parámetros pero se mantienen fijos los términos residuales, es decir, las características inobservables, como podría ser una habilidad particular de los individuos, no se modifican, y tampoco su retribución. Esta simulación se realiza en forma separada para la educación y experiencia con el fin de aislar ambos efectos.

Finalmente, las características inobservables que afectan el salario de las mujeres se modelan como el término de error de las ecuaciones de salario estimadas. A los efectos de simular una nueva distribución de ingresos y capturar los efectos producidos por cambios en las características inobservables de las mujeres, se estima el efecto multiplicando el término de error de la ecuación de salarios de $t$ por el cociente $\left(\sigma_{t} / \sigma_{t^{\prime}}\right)$, donde $\sigma_{t}$ y $\sigma_{t^{\prime}}$ son los desvíos estimados para los residuos de la ecuación de salarios de cada período. Los términos residuales de las regresiones son los que permiten estimar las características inobservables de las mujeres. Sin embargo, para aquellas mujeres que no trabajan no se cuenta con el verdadero valor de su salario y, por lo tanto, no es posible estimar el valor de sus inoblervables al no tener un residuo. En este caso, como fue mencionado anteriormente, se sigue la literatura sobre microdescomposiciones y se asignan errores simulados consistentemente con su situación laboral original.

Debido a que la posición que ocupan los individuos dentro del hogar afecta sus decisiones de empleo, siguiendo a Bourguigon et al. (1998), la muestra se parte en tres grupos y se estima en forma separada para jefas, cónyuges y otras. Si bien la mayoría de las variables explicativas contempladas en cada uno de los modelos coinciden, se incorporan variables particulares para cada caso. De este modo, para cada uno de estos grupos, y para cada año, se estima una ecuación de salarios y otra de oferta laboral.

A los efectos de medir los retornos a la educación, en todos los modelos utilizados para estimar salarios se incluyeron variables binarias que indican el máximo nivel alcanzado por las mujeres, omitiendo en las estimaciones la categoría de primaria incompleta con el fin de evitar problemas de multicolinealidad. Por otra parte, con el objetivo de estimar los retornos a la experiencia de incorporan como variables explicativas la edad y la edad 
al cuadrado de las mujeres. En Uruguay aproximadamente la mitad de las personas que viven en zonas urbanas lo hacen en la capital del país, a los efectos de controlar por diferencias originadas por el lugar de residencia, se incorpora una variable dummy que adopta el valor uno cuando la persona reside en Montevideo.

Como fue mencionado, existen diferencias en las decisiones de empleo originadas por la posición que ocupan las mujeres en el hogar. En este sentido, en el caso de las jefas y cónyuges se incorpora la variable número de hijos, mientras que no se cuenta con dicha información para otras. Asimismo, para cónyuges y otras se toma en consideración el status laboral del jefe del hogar al momento de determinar la entrada al mercado laboral. En el caso de jefas y otras se incorpora cómo variable independiente si la mujer se encuentra casada o en unión libre. Para todas las ecuaciones de selección se incorporan como variables explicativas el máximo nivel educativo alcanzado, la edad y edad al cuadrado, si asiste o no a un centro de enseñanza y el ingreso laboral masculino del hogar al que pertenecen.

Se consideran los efectos en el empleo generados por las mujeres de entre 14 y 64 años de edad. La restricción etaria se debe a que en Uruguay se considera a la población en edad de trabajar a los mayores de 13 años, y porque luego de los 64 años la mayor parte de mujeres ya se ha retirado del mercado de trabajo. Con el fin de evitar introducir efectos producidos por variaciones en los precios, se trabaja con valores a precios constantes a diciembre de 2012 .

\section{Resultados}

En este apartado se presentan los resultados obtenidos a partir del trabajo de descomposición realizado con el fin de evaluar el posible efecto del empleo femenino sobre la distribución del ingreso.

Como fue mencionado, debido a las diferencias de las decisiones de participación de las mujeres en función del lugar que ocupan dentro del hogar, las ecuaciones de ingresos horarios (8) y de horas trabajadas (9) se estiman en forma separada para jefas, cónyuges y otras. En las Tablas A2, A3 y A4 del Apéndice se reportan las estimaciones del modelo de ingresos horarios para jefas, cónyuges y otras, respectivamente, mientras que en las Tablas A5, A6 y A7 se presentan los resultados de las estimaciones de los modelos de horas trabajadas para dichos grupos de mujeres. A continuación, se describen algunos fenómenos que interesa destacar a partir de los resultados de las estimaciones mencionadas.

En lo que respecta a la evolución de los ingresos horarios, se observa que la experiencia, aproximada por la edad de las mujeres, tiene un efecto estadísticamente significativo sobre los ingresos horarios que, si bien es positivo, decrece con la edad, adoptando un valor mayor del coeficiente en el caso de otras respecto a jefas y cónyuges. Por otra parte, independientemente de la posición que ocupen en el hogar y del año analizado, los resultados de la estimación del logaritmo del ingreso horario muestran que, los coeficientes que miden los retornos a la educación son positivos y, en casi todos los casos, significativos al 1\%. Al mismo tiempo, los resultados arrojan que a mayor nivel educativo mayor el 
diferencial salarial. Sin embargo, si bien entre 1991 y 2000 los premios a la educación de las jefas y otras aumentaron, entre 2000 y 2012 los retornos a la educación de estos dos grupos de mujeres se redujeron. Por su parte, las cónyuges, en términos generales, tuvieron una caída en sus retornos entre 1991 y 2000 y un aumento entre 2000 y 2012. Una caída de los retornos a la educación sugeriría una caída de los ingresos de las más educadas en términos relativos, lo cual produciría una disminución de la desigualdad. El efecto final dependerá del peso de cada grupo en el total.

Una vez realizadas las estimaciones de los parámetros se implementa la metodología descripta en la sección anterior. El cambio de la participación de la mujer en el mercado de trabajo mediante cambios en el empleo se evalúa sobre la pobreza y la desigualdad. En el primero de los casos, se estiman variaciones en el indicador FGT (Foster, Greer y Thorbecke, 1984) con parámetro 0, es decir, incidencia de la pobreza, considerando una línea de pobreza per cápita de 4 dólares por día a PPA. Adicionalmente, se estiman los efectos sobre la brecha de pobreza y la llamada profundidad de la pobreza, FGT(1) y FGT(2) respectivamente, cuyos resultados pueden observarse en las Tablas A8 y A9 del Apéndice. Con el fin de estimar los efectos sobre la desigualdad se utiliza el índice de Gini. Todos los indicadores se computan sobre el ingreso per cápita familiar.

Debido a que los resultados de las descomposiciones dependen del año que se considere como punto de partida, los resultados obtenidos en este trabajo surgen de promedios elaborados al alternar los años que se comparan como base. Para otorgarle robustez a los resultados obtenidos se emplean técnicas de bootstrap con 200 repeticiones.

En las Tablas 5 y 6 se presentan los resultados de la descomposición para la desigualdad y la pobreza, respectivamente. La forma en la que deben leerse dichas tablas es la siguiente. Un valor positivo indica que hubo un aumento en el indicador considerado, mientras que un valor negativo indica que hubo una caída, ya sea de la pobreza o de la desigualdad. Además del signo de la variación debe observarse la magnitud de la misma. De esta forma, se podrán realizar valoraciones respecto a la importancia relativa que cada efecto tuvo sobre la distribución del ingreso.

Los efectos de las decisiones de empleo de las mujeres sobre la desigualdad se reportan en forma resumida en la Tabla 5, donde se muestran las variaciones en el coeficiente de Gini. Entre 1991 y 2000, el índice de Gini estimado sobre el ingreso per cápita familiar (IPCF) aumentó 2.95 puntos. De acuerdo con las microsimulaciones realizadas, el efecto empleo sobre la desigualdad fue de -0.24, indicando que, ceteris paribus, la concentración del ingreso hubiese caído como resultado del aumento del empleo femenino, a diferencia de lo que efectivamente se observó. Al distinguir entre el efecto estado y el efecto horas, se encuentra que el primero, prácticamente, no tuvo incidencia sobre la desigualdad (-0.02), mientras el efecto horas puede atribuirse una disminución del indicador de 0.20.

Por otra parte, entre 2000 y 2012, el índice de Gini sufrió una caída de 2.87 puntos. Si bien la contribución del efecto estado y el de horas trabajadas no representa una gran proporción de la misma, ambos son de signo negativo y significativo del punto de vista estadístico en el $95 \%$ y $99 \%$ de los casos, respectivamente. Al considerar el efecto empleo agregado, se observa que este se ubica en -0.25 , siendo superior a los efectos considerados de forma aislada. En esta oportunidad el efecto estado resulta algo más igualador que 
en el sub-período anterior. Ello podría deberse al mayor aumento de la tasa de empleo femenina y, en particular, a la mayor variación del empleo de las mujeres pertenecientes a los hogares más pobres.

Finalmente, al estudiar todo el período bajo análisis, se encuentra que la desigualdad se mostró casi idéntica entre 1991 y 2012. Sin embargo, la contribución del empleo femenino a la desigualdad, tanto del efecto estado como del efecto horas, entre los dos extremos del período fue siempre negativa, de -0.13 y -0.41 a un nivel de significación estadística de $5 \%$ y $1 \%$, respectivamente.

Tabla 5:

Descomposición del cambio en la desigualdad medida por el índice de GINI: Uruguay 1991-2012.

\begin{tabular}{lccc}
\hline & \multicolumn{3}{c}{ Valor del indicador } \\
& 1991 & 2000 & 2012 \\
\hline Gini & 41.42 & 44.37 & 41.50 \\
\hline & $1991-2000$ & $2000-2012$ & $1991-2012$ \\
\hline Variación observada & 2.95 & -2.87 & 0.08 \\
Efectos & & & \\
\hline Empleo & $-0.24^{* * *}$ & $-0.25^{* * *}$ & $-0.54^{* * *}$ \\
Estado & -0.02 & $-0.06^{* *}$ & $-0.13^{* *}$ \\
Horas trabajadas & $-0.20^{* * *}$ & $-0.21^{* * *}$ & $-0.41^{* * *}$ \\
\hline Retornos a la educación & -0.18 & -0.17 & $-0.39^{* * *}$ \\
Retornos a la experiencia & -0.09 & 0.08 & 0.01 \\
Inobservables & 0.02 & $0.15^{* *}$ & $0.16^{* *}$ \\
\hline \hline
\end{tabular}

Fuente: Elaboración propia en base a ECH.

Nota: $* * *$ significativo al $1 \%, * *$ significativo al $5 \%, *$ significativo al $10 \%$. Localidades urbanas con más de 5000 hab.

Por su parte, en lo que respecta a los efectos de los retornos, mientras los cambios en los premios a la educación tuvieron un efecto pequeño e igualador en todos los períodos analizados, los retornos a la experiencia (con excepción de 1991-2000) y los inobservables, o bien fueron neutrales o desigualadores. En el caso de los retornos a la experiencia, los efectos encontrados no fueron significativos del punto de vista estadístico en ningún caso.

En lo referente a la tasa de incidencia de la pobreza, al distinguir entre el efecto estado y el de las horas trabajadas, el ejercicio permite evaluar la importancia relativa que tuvo cada uno sobre el indicador FGT(0). Como puede apreciarse, entre 1991 y 2000 la proporción de individuos por debajo de la línea de pobreza se incrementó en 0.46 puntos. Ahora bien, el efecto estado de las mujeres contribuyó en reducir la misma en 0.14 puntos, lo cual significa que si solo los parámetros que guían las decisiones (posibilidades) binarias de empleo de las mujeres hubiesen cambiado entre esos dos años, mientras los demás elementos se hubiesen mantenido constantes, entonces la tasa de pobreza se hubiese reducido. Es decir, el incremento de la pobreza durante la década del noventa parece no haberse originado en los cambios en las decisiones de estatus ocupacional de las mujeres. En el mismo sentido, pero con mayor peso, los cambios en la cantidad de horas promedio 
Tabla 6:

Descomposición del cambio en la tasa de incidencia de la pobreza: Uruguay 1991-2012.

\begin{tabular}{lccc}
\hline & \multicolumn{3}{c}{ Valor del indicador } \\
& 1991 & 2000 & 2012 \\
\hline FGT(0) & 10.76 & 11.22 & 8.26 \\
\hline & $1991-2000$ & $2000-2012$ & $1991-2012$ \\
\hline Variación observada & 0.46 & -2.96 & -2.50 \\
Efectos & & & \\
\hline Empleo & $-0.48^{* * *}$ & $-0.53^{* * *}$ & $-1.00^{* * *}$ \\
Estado & $-0.14^{* *}$ & $-0.21^{* * *}$ & $-0.39^{* * *}$ \\
Horas trabajadas & $-0.38^{* * *}$ & $-0.46^{* * *}$ & $-0.80^{* * *}$ \\
\hline Retornos a la educación & -0.07 & -0.27 & -0.25 \\
Retornos a la experiencia & 0.35 & -1.00 & -0.91 \\
Inobservables & -0.03 & $-0.11^{* *}$ & $-0.16^{* * *}$ \\
\hline \hline
\end{tabular}

Fuente: Elaboración propia en base a ECH.

Nota: $* * *$ significativo al $1 \%, * *$ significativo al $5 \%,{ }^{*}$ significativo al $10 \%$. Localidades urbanas con más de 5000 hab.

trabajas por las mujeres contribuyeron a reducir la pobreza. Concretamente, si los únicos cambios entre 1991 y 2000 hubiesen sido en los parámetros que determinan la cantidad de horas trabajadas por las mujeres, la pobreza se hubiese reducido 0.38 puntos en lugar de haber aumentado. Asimismo, el efecto empleo se ubicó en -0.48, contribuyendo a una caída del indicador. Este efecto fue de una magnitud casi idéntica, pero de signo contrario, a la variación efectivamente observada en la tasa de pobreza.

Para el período 2000-2012 los resultados son algo diferentes. La variación de la tasa de indecencia de la pobreza observada fue negativa, pasando del $11.22 \%$ a $8.26 \%$ de los individuos. Tanto el efecto empleo como el de horas trabajadas contribuyeron a la reducción del indicador. Esto podría estar vinculado a un resultado visto anteriormente, donde se podía observar que durante dicho período las mujeres pertenecientes a hogares más pobres fueron las que se incorporaron en mayor medida al mercado de trabajo (Figura 4). Por su parte, el efecto empleo fue de -0.53, siendo el más importante de los efectos estimados y colaborando con caída de la tasa de pobreza, pero en menor medida que la simple suma de los efectos horas y estado.

Entre 1991 y 2012 la pobreza en Uruguay disminuyó aproximadamente 23\%, lo cual fue resultado de múltiples factores. Los cambios en las decisiones de empleo de las mujeres parecen haber formado parte de dicho proceso. De acuerdo con el ejercicio, el aumento de la cantidad de mujeres ocupadas y los cambios en las horas trabajadas contribuyeron a la reducción de la incidencia de la pobreza a lo largo de todo el período, arrojando efectos estadísticamente significativos. En particular, la magnitud del efecto empleo fue equivalente al $40 \%$ de la caída de la tasa de pobreza observada.

Por otra parte, los efectos producidos por los cambios en los retornos a la educación, si bien no fueron grandes en términos relativos ni absolutos, contribuyeron a reducir la pobreza en todos los períodos estudiados. A los retornos a la experiencia de las mujeres 
le es atribuible un aumento de la pobreza de 0.35 entre 1991 y 2000 y una reducción de 1.00 entre 2000 y 2012. Este efecto, junto con el efecto horas, fue el más importante de los estudiados en términos relativos al momento de explicar la reducción de la pobreza entre 1991 y 2012. Sin embargo, ni el efecto producido por los cambios en los retornos a la educación, ni el producido por cambios en los retornos a la eperiencia sobre la pobreza, resultaron significativos para los períodos estudiados. Finalmente, la incidencia de los inobservables fue no significativa y de -0.03 entre 1991 y 2000 , significativa y de -0.11 entre 2000 y 2012 y significativa y de -0.16 cuando se considera el período completo.

En las Tablas A8 y A9 del Apéndice se reportan los resultados de computar la brecha de la pobreza -el FGT(1) - y la profundidad de la misma -el FGT(2)- para los distintos períodos. Los resultados encontrados, si bien presentan alguna diferencia, van en la misma línea que lo descrito para la tasa de incidencia de la pobreza - FGT(0). De todas formas, con excepción del efecto horas entre 1991 y 2012 para el caso del FGT(1), todos los resultados resultan no significativos.

Los diferentes efectos observados entre 1991, 2000 y 2012, parecen estar relacionados con el perfil de las mujeres que se han ido incorporando al mercado laboral. Durante los primeros años, el aumento del empleo femenino estuvo impulsado en mayor medida por la incorporación al mercado laboral de mujeres pertenecientes a los hogares de mayores ingresos y de mayor nivel educativo. El resultado de dicho fenómeno fue un efecto menor sobre la distribución del ingreso y la pobreza.

Por otra parte, una vez que estas mujeres se incorporaron al mercado laboral, aumentó la posibilidad de incorporación de las menos educadas y, de esta forma, entre 2000 y 2012, se pudo observar un importante aumento de la tasa de empleo de las mujeres pertenecientes a los hogares más pobres. De este modo, la caída de la desigualdad y, en particular, de la pobreza, tienen una mayor contribución de los efectos empleo y horas, como resultado de un aumento de los ingresos de los hogares más pobres, donde un importante número de mujeres dejan de tener ingresos cero. 


\section{Conclusiones}

A lo largo del período comprendido entre 1991 y 2012 la pobreza y la desigualdad en Uruguay sufrieron importantes variaciones. En un primer momento experimentaron un período de crecimiento, para luego revertir dicha tendencia y comenzar a disminuir. Al comparar los dos extremos del período estudiado, se encuentra que mientras la pobreza disminuyó 2.5 puntos porcentuales (aproximadamente $23 \%$ ), la desigualdad se mantuvo prácticamente estable, mostrando una variación positiva de 0.08 puntos (variación inferior a $1 \%$ ). Estos cambios se dieron en un contexto en el cual las mujeres aumentaron considerablemente su nivel de empleo, pasando de tasas promedio inferiores al $40 \%$ a superar el $50 \%$. En particular, las mujeres de entre 14 y 64 años de edad, que son las consideradas en la simulación, pasaron de registrar una tasa de empleo de $47.1 \%$ a $61.5 \%$. En este trabajo se estudia desde el punto de vista empírico, cuál es la relación entre los cambios en el mercado de trabajo, en particular las modificaciones en el empleo de las mujeres, y los cambios ocurridos en la distribución del ingreso. El análisis se realiza para todo el período 1991-2012 y se distingue además lo ocurrido entre 1991-2000 de 2000-2012.

Se optó por abordar el estudio utilizando la metodología de microdescomposición propuesta por Bourguignon, Ferreira y Lustig (1998). Esta metodología permite distinguir, además del efecto agregado del cambio del empleo femenino, entre el efecto generado por cambios en las decisiones de estatus de empleo (si trabaja o no) de los producidos por modificaciones en las horas trabajadas. Los efectos se estiman sobre indicadores usuales de pobreza y concentración del ingreso, FGT e índice de Gini. Se emplean técnicas de bootstrap con el fin de dar robustez a las estimaciones realizadas.

Si bien los cambios en el empleo femenino no parecen ser el principal determinante cuando se busca explicar la evolución de la distribución del ingreso, se puede establecer que han tenido efectos significativos del punto de vista estadístico e importantes en términos relativos. En primer lugar, los resultados muestran que los cambios en empleo femenino contribuyeron en todo momento a reducir los niveles de pobreza. En segundo lugar, en lo que respecta a la desigualdad, se observó que el aumento del empleo de las mujeres en un primer momento tuvo resultados modestos sobre la desconcentración del ingreso, y más acentuados hacia el final del período. En ambos casos, el efecto horas fue más importante al momento de explicar la caída de los indicadores de distribución que el efecto estado y, además, fue más importante que el resto de los efectos estimados en forma aislada, tanto en términos de magnitud como de significatividad estadística.

Al distinguir entre 1991-2000 y 2000-2012 se puede observar que, mientras en el primer sub-período estudiado la pobreza y la desigualdad aumentan, en el sub-período siguiente se produce la evolución inversa. Sin embargo, la contribución estimada del empleo femenino sobre estos aspectos de la distribución del ingreso se mantuvo en la misma dirección. Es decir, el signo de los efectos estimados fue idéntico a lo largo de todo el período de estudio. 


\section{Referencias bibliográficas}

Alves, G., Amarante, V., Salas, G. y Vigorito, A. (2012), "La desigualdad del ingreso en Uruguay entre 1986 y 2009". [En Línea] Serie Documentos de Trabajo, D.T. 03/12. Instituto de Economía, disponible en: http://www.iecon.ccee.edu.uy/dt-03-12-ladesigualdad-del-ingreso-en-uruguay-entre-1986-y-2009/publicacion/286/es/.

Amemiya, T. (1985). Advanced Econometrics. Harvard University Press.

Blinder, A. (1973). "Wage discrimination: reduced form and structural estimate". The Journal of Human Resources. Volumen 8. Otoño, 1973, pp 436-455.

Bourguignon, F., Ferreira, F. y Lustig, N. (1998). The microeconomics of income distribution dynamics in East Asia and Latin America. IDB-World Bank Research Proposal.

Chioda, L. (2011). Work and Family, Latin American and Caribbean Women in Search of a New Balance. Banco Mundial. Washington, DC. Conference Edition.

Espino, A. (2003), "El aporte de las remuneraciones femeninas en los hogares y sus efectos enla distribución del ingresos". [En Línea] Serie Documentos de Trabajo, D.T. 4/03. Instituto de Economía, disponible en:

http://www.iecon.ccee.edu.uy/dt-04-03-el-aporte-de-las-remuneraciones-femeninas-en-loshogares-y-sus-efectos-en-la-distribucion-del-ingreso/publicacion/88/es/.

Espino, A. y Leites. M. (2008). "Oferta laboral femenina en Uruguay: evolución e implicancias: 1981 -2006". [En Línea] Serie Documentos de Trabajo, D.T. 07/08. Instituto de Economía, disponible en: http://www.iecon.ccee.edu.uy/dt-07-08-oferta-laboralfemenina-en-uruguay-evolucion-e-implicancias-1981-2006/publicacion/104/es/.

Fortin, N., Lemieux, T. y Firpo, S. (2011). "Decomposition Method in Economics", en Ashenfelter, O. y Card, D. (eds.), Handbook of Labor Economics. Elsevier, edición 1, volumen 4 , número 4.

Gasparini, L. (coord.), Acosta, P., Bebczuk, R., Golla, J., Marchionni, M., Margot, D., Mezza, A., Porto, G. y Sosa Escudero, W. (2000). "Determinantes de la distribución del ingreso". Cuadernos de Economía No 54. Convenio Ministerio de Economía de la Provincia de Buenos Aires-Facultad de Ciencias Económicas, Universidad Nacional de La Plata.

Gasparini, L., Marchionni, M. y Sosa Escudero, W. (2004). "Characterization of inequality changes through microeconometric decompositions. The case of Greater Buenos Aires". En Bourguignon, F., Ferreira, F. y Lustig, N. (eds.), The Microeconomics of Income Distribution Dynamics in East Asia and Latin America. New York, Oxford University Press, pp. 47-82.

Gónzalez, C. y Rossi, M. (2003), "Participación femenina en el mercado de trabajo: efectos sobre la distribución del ingreso en Uruguay". [En Línea] Serie de Documentos de Trabajo, DT 12/03. Departamento de Economía Facultad de Ciencias Sociales, disponible en: http://decon.edu.uy/publica/2003/Doc1203.pdf. 
Killingsworth, M. y Heckman, J. (1986). "Female Labor Supply: A Survey". en Orley Ashenfelter and Richard Layard (eds.), Handbook of Labor Economics. Volumen 1, pp. 103-204.

Juhn, C., Murphy, K. y Pierce, B. (1993). "Wage inequality and the rise in returns to skill". en Journal of Political Economy. Volumen 101, número 3. Junio 1993, pp 410-442.

Marroig, A. y Oreriro, C. (2008). "Determinantes de la distribución del ingreso en Uruguay 1991-2005. Un análisis de microsimulaciones". en Revista Quantum. Volumen III, número 2. Diciembre 2008, pp 46-63.

Oaxaca, R. (1973). "Male-female wage diferentials in urban labor market". en International Economic Review. Volumen 14. Octubre 1973, pp 693-709.

Vigorito, A., (1999). "Una distribución del ingreso estable. El caso de Uruguay 1986 1997". [En Línea] Serie Documentos de Trabajo, D.T. 6/99. Instituto de Economía, disponible en: http://www.iecon.ccee.edu.uy/dt-06-99-una-distribucion-del-ingreso-estableel-caso-de-uruguay-1986-1997/publicacion/101/es/ .

Zoloa, J., (2008). Los cambios en la distribución del ingreso de Argentina entre 1998 y 2005: Un análisis de microdescomposiciones utilizando información de paneles. Tesis de Maestría en Economía. Argentina, Uiversidad Nacional de La Plata. 


\section{A. Apéndice}

Tabla A1. Variables agregadas.

\begin{tabular}{lccc}
\hline \hline & $\mathbf{1 9 9 1}$ & $\mathbf{2 0 0 0}$ & $\mathbf{2 0 1 2}$ \\
\hline PIB per cápita en U\$S & 3,720 & 6,914 & 14,767 \\
Tasa de actividad & & & \\
Total & 54.4 & 59.6 & 64.0 \\
Hombres & 73.3 & 71.9 & 73.0 \\
Mujeres & 43.8 & 49.1 & 56.2 \\
Tasa de empleo & & & \\
Total & 52.3 & 51.5 & 59.7 \\
Hombres & 68.2 & 64.1 & 69.1 \\
Mujeres & 38.7 & 40.8 & 51.5 \\
Tasa de desempleo & & & \\
Total & 8.9 & 13.6 & 6.8 \\
Hombres & 7.0 & 10.9 & 5.3 \\
Mujeres & 11.5 & 17.0 & 8.3 \\
FGT(0) & 10.8 & 11.2 & 8.3 \\
FGT(1) & 3.3 & 3.3 & 2.4 \\
FGT(2) & 1.5 & 1.5 & 1.0 \\
GINI & 41.4 & 44.4 & 41.5 \\
\hline \hline
\end{tabular}

Fuente: Elaboración propia en base a datos de INE y BCU.

Nota: La información sobre mercado de trabajo son datos oficiales para personas mayores de 13 años pertenecientes a localidades urbanas con más de 5000 habitantes. 
Tabla A2. Estimación de la ecuación de ingresos horarios para jefas de hogar. Método de Heckman Máximo Verosímil.

Variable dependiente: logaritmo del ingreso laboral horario.

\begin{tabular}{|c|c|c|c|}
\hline & 1991 & 2000 & 2012 \\
\hline \multirow[t]{2}{*}{ Edad } & $0.0686^{* * *}$ & $0.0340^{* *}$ & $0.0387^{* * *}$ \\
\hline & {$[0.0175]$} & {$[0.0152]$} & {$[0.00563]$} \\
\hline \multirow[t]{2}{*}{ Edad2 } & $-0.000805^{* * *}$ & -0.000284 & $-0.000358^{* * *}$ \\
\hline & {$[0.000207]$} & {$[0.000183]$} & {$[6.65 \mathrm{e}-05]$} \\
\hline \multirow[t]{2}{*}{ Pric } & $0.117^{* *}$ & $0.189 * * *$ & 0.0456 \\
\hline & {$[0.0561]$} & {$[0.0599]$} & {$[0.0405]$} \\
\hline \multirow[t]{2}{*}{ Seci } & $0.461^{* * *}$ & $0.496^{* * *}$ & $0.337^{* * *}$ \\
\hline & {$[0.0578]$} & {$[0.0586]$} & {$[0.0392]$} \\
\hline \multirow[t]{2}{*}{ Secc } & 0.339 & $0.759 * * *$ & $0.667 * * *$ \\
\hline & {$[0.343]$} & {$[0.0973]$} & {$[0.0434]$} \\
\hline \multirow[t]{2}{*}{ Supi } & $0.651^{* * *}$ & $0.959^{* * *}$ & $0.827 * * *$ \\
\hline & {$[0.0863]$} & {$[0.0740]$} & {$[0.0449]$} \\
\hline \multirow[t]{2}{*}{ Supc } & $1.067^{* * * *}$ & $1.316^{* * *}$ & $1.225^{* * *}$ \\
\hline & {$[0.0770]$} & {$[0.0706]$} & {$[0.0430]$} \\
\hline \multirow[t]{2}{*}{ Montevideo } & $0.377^{* * *}$ & $0.367^{* * *}$ & $0.138^{* * *}$ \\
\hline & {$[0.0389]$} & {$[0.0359]$} & {$[0.0149]$} \\
\hline \multirow[t]{2}{*}{ Constante } & $2.160^{* * *}$ & $2.749^{* * *}$ & $2.860 * * *$ \\
\hline & {$[0.393]$} & {$[0.338]$} & {$[0.131]$} \\
\hline \multicolumn{4}{|c|}{ Ecuación de selección } \\
\hline \multirow[t]{2}{*}{ Edad } & $0.188^{* * *}$ & $0.264^{* * *}$ & $0.198^{* * *}$ \\
\hline & {$[0.0252]$} & {$[0.0172]$} & {$[0.00913]$} \\
\hline \multirow[t]{2}{*}{ Edad2 } & $-0.00260^{* * *}$ & $-0.00341^{* * *}$ & $-0.00256^{* * *}$ \\
\hline & {$[0.000266]$} & {$[0.000194]$} & {$[0.000106]$} \\
\hline \multirow[t]{2}{*}{ Pric } & $0.203^{* * *}$ & -0.00159 & $0.118^{*}$ \\
\hline & {$[0.0775]$} & {$[0.0818]$} & {$[0.0620]$} \\
\hline \multirow[t]{2}{*}{ Seci } & $0.346^{* * *}$ & $0.205^{* *}$ & $0.353^{* * *}$ \\
\hline & {$[0.0819]$} & {$[0.0813]$} & {$[0.0590]$} \\
\hline \multirow[t]{2}{*}{ Secc } & 6.961 & $0.474^{* * *}$ & $0.611^{* * *}$ \\
\hline & [65011] & {$[0.175]$} & {$[0.0704]$} \\
\hline \multirow[t]{2}{*}{ Supi } & $0.528 * * *$ & $0.351^{* * *}$ & $0.604^{* * *}$ \\
\hline & {$[0.172]$} & {$[0.124]$} & {$[0.0780]$} \\
\hline \multirow[t]{2}{*}{ Supc } & $0.819^{* * *}$ & $0.849 * * *$ & $0.939 * * *$ \\
\hline & {$[0.134]$} & [0.110] & {$[0.0686]$} \\
\hline \multirow[t]{2}{*}{ Casado } & $-0.507^{* * *}$ & $-0.221^{* * *}$ & $-0.357 * * *$ \\
\hline & {$[0.128]$} & {$[0.0739]$} & {$[0.0359]$} \\
\hline \multirow[t]{2}{*}{ Asiste } & $-0.0785^{* *}$ & $-0.163^{* * *}$ & $-0.172^{* * *}$ \\
\hline & {$[0.0319]$} & {$[0.0243]$} & {$[0.0148]$} \\
\hline \multirow[t]{2}{*}{ Estado jefe } & $-0.442^{*}$ & -0.203 & 0.0228 \\
\hline & {$[0.237]$} & {$[0.147]$} & {$[0.0745]$} \\
\hline \multirow[t]{2}{*}{ ILHH } & $-6.20 \mathrm{e}-06^{* *}$ & $-2.98 \mathrm{e}-06$ & $-1.62 \mathrm{e}-06$ \\
\hline & {$[2.77 \mathrm{e}-06]$} & {$[2.02 \mathrm{e}-06]$} & [1.13e-06] \\
\hline \multirow[t]{2}{*}{ Constante } & $-2.376^{* * *}$ & $-4.002^{* * *}$ & $-2.721^{* * *}$ \\
\hline & [0.593] & {$[0.371]$} & [0.194] \\
\hline Observaciones & 2192 & 2984 & 10513 \\
\hline
\end{tabular}

Fuente: Elaboración propia en base a datos de ECH.

Nota: Errores estándar entre paréntesis. ${ }^{* *}$ significativo al $1 \%,{ }^{* *}$ significativo al $5 \%,{ }^{*}$ significativo al $10 \%$. 
Tabla A3. Estimación de la ecuación de ingresos horarios para cónyuges. Método de Heckman Máximo Verosímil.

Variable dependiente: logaritmo del ingreso laboral horario.

\begin{tabular}{|c|c|c|c|}
\hline & 1991 & 2000 & 2012 \\
\hline \multirow[t]{2}{*}{ Edad } & $0.0383^{* * *}$ & $0.0731^{* * *}$ & $0.0414^{* * *}$ \\
\hline & [0.0101] & {$[0.00945]$} & {$[0.00540]$} \\
\hline \multirow[t]{2}{*}{ Edad2 } & $-0.000381^{* * *}$ & $-0.000812^{* * *}$ & $-0.000400^{* * *}$ \\
\hline & {$[0.000124]$} & {$[0.000115]$} & {$[6.45 \mathrm{e}-05]$} \\
\hline \multirow[t]{2}{*}{ Pric } & $0.144^{* * *}$ & 0.0106 & $0.195^{* * *}$ \\
\hline & {$[0.0364]$} & {$[0.0495]$} & {$[0.0508]$} \\
\hline \multirow[t]{2}{*}{ Seci } & $0.476^{* * *}$ & $0.320 * * *$ & $0.430 * * *$ \\
\hline & {$[0.0369]$} & {$[0.0496]$} & {$[0.0499]$} \\
\hline \multirow[t]{2}{*}{ Secc } & $0.864^{* * *}$ & $0.700 * * *$ & $0.826^{* * *}$ \\
\hline & {$[0.137]$} & {$[0.0686]$} & {$[0.0537]$} \\
\hline \multirow[t]{2}{*}{ Supi } & $0.830^{* * *}$ & $0.811^{* * *}$ & $0.955^{* * *}$ \\
\hline & {$[0.0587]$} & {$[0.0636]$} & {$[0.0562]$} \\
\hline \multirow[t]{2}{*}{ Supc } & $1.214^{* * *}$ & $1.258^{* * *}$ & $1.417^{* * *}$ \\
\hline & {$[0.0592]$} & {$[0.0645]$} & {$[0.0553]$} \\
\hline \multirow[t]{2}{*}{ Montevideo } & $0.303^{* * *}$ & $0.313^{* * *}$ & $0.150^{* * *}$ \\
\hline & {$[0.0222]$} & {$[0.0232]$} & {$[0.0141]$} \\
\hline \multirow[t]{2}{*}{ Constante } & $2.600 * * *$ & $2.093^{* * *}$ & $2.630^{* * *}$ \\
\hline & {$[0.238]$} & {$[0.221]$} & {$[0.131]$} \\
\hline \multicolumn{4}{|c|}{ Ecuación de selección } \\
\hline \multirow[t]{2}{*}{ Edad } & $0.181^{* * *}$ & $0.195^{* * *}$ & $0.166^{* * *}$ \\
\hline & {$[0.00956]$} & {$[0.00999]$} & {$[0.00788]$} \\
\hline \multirow[t]{2}{*}{ Edad2 } & $-0.00236^{* * *}$ & $-0.00247^{* * *}$ & $-0.00210^{* * *}$ \\
\hline & {$[0.000115]$} & {$[0.000120]$} & {$[9.44 \mathrm{e}-05]$} \\
\hline \multirow[t]{2}{*}{ Pric } & -0.0229 & $0.0909 *$ & $0.300^{* * *}$ \\
\hline & {$[0.0400]$} & {$[0.0544]$} & {$[0.0627]$} \\
\hline \multirow[t]{2}{*}{ Seci } & $0.198 * * *$ & $0.380 * * *$ & $0.525 * * *$ \\
\hline & {$[0.0409]$} & {$[0.0535]$} & {$[0.0605]$} \\
\hline \multirow[t]{2}{*}{ Secc } & $0.344^{*}$ & $0.774^{* * *}$ & $0.936^{* * *}$ \\
\hline & [0.181] & {$[0.0866]$} & {$[0.0678]$} \\
\hline \multirow[t]{2}{*}{ Supi } & $0.664^{* * *}$ & $0.711^{* * *}$ & $0.945^{* * *}$ \\
\hline & {$[0.0781]$} & {$[0.0810]$} & {$[0.0769]$} \\
\hline \multirow[t]{2}{*}{ Supc } & $1.303^{* * *}$ & $1.504^{* * *}$ & $1.562^{* * *}$ \\
\hline & {$[0.0653]$} & {$[0.0730]$} & {$[0.0705]$} \\
\hline \multirow[t]{2}{*}{ Casado } & $-0.0947 * * *$ & $-0.112^{* * *}$ & $-0.133^{* * *}$ \\
\hline & {$[0.0120]$} & {$[0.0131]$} & {$[0.0120]$} \\
\hline \multirow[t]{2}{*}{ Asiste } & 0.107 & 0.0106 & -0.00781 \\
\hline & {$[0.106]$} & {$[0.0991]$} & {$[0.0669]$} \\
\hline \multirow[t]{2}{*}{ Estado jefe } & -0.0296 & $0.116^{* *}$ & $0.234^{* * *}$ \\
\hline & {$[0.0445]$} & {$[0.0465]$} & [0.0416] \\
\hline \multirow[t]{2}{*}{ ILHH } & $-1.38 \mathrm{e}-06^{* * *}$ & $-4.21 \mathrm{e}-06^{* * *}$ & $-5.72 \mathrm{e}-06^{* * *}$ \\
\hline & {$[3.80 \mathrm{e}-07]$} & [6.23e-07] & {$[5.85 \mathrm{e}-07]$} \\
\hline \multirow[t]{2}{*}{ Constante } & $-3.292^{* * *}$ & $-3.777^{* * *}$ & $-3.211^{* * *}$ \\
\hline & [0.188] & [0.200] & [0.163] \\
\hline Observaciones & 9432 & 8106 & 12669 \\
\hline
\end{tabular}

Fuente: Elaboración propia en base a datos de ECH.

Nota: Errores estándar entre paréntesis. ${ }^{* *}$ significativo al $1 \%,{ }^{* *}$ significativo al $5 \%,{ }^{*}$ significativo al $10 \%$. 
Tabla A4. Estimación de la ecuación de ingresos horarios para otras mujeres del hogar.

Método de Heckman Máximo Verosímil.

Variable dependiente: logaritmo del ingreso laboral horario.

\begin{tabular}{|c|c|c|c|}
\hline & 1991 & 2000 & 2012 \\
\hline \multirow[t]{2}{*}{ Edad } & $0.0735^{* * *}$ & $0.0726^{* * *}$ & $0.0465^{* * *}$ \\
\hline & {$[0.00931]$} & {$[0.0102]$} & {$[0.00834]$} \\
\hline \multirow[t]{2}{*}{ Edad2 } & $-0.000861^{* * *}$ & $-0.000772^{* * *}$ & $-0.000449^{* * *}$ \\
\hline & {$[0.000124]$} & [0.000133] & [0.000107] \\
\hline \multirow[t]{2}{*}{ Pric } & 0.0963 & $0.295^{* * *}$ & 0.0607 \\
\hline & {$[0.0592]$} & {$[0.0729]$} & {$[0.0917]$} \\
\hline \multirow[t]{2}{*}{ Seci } & $0.372^{* * *}$ & $0.543^{* * *}$ & $0.305^{* * *}$ \\
\hline & {$[0.0586]$} & {$[0.0713]$} & {$[0.0904]$} \\
\hline \multirow[t]{2}{*}{ Secc } & $0.741^{* * *}$ & $0.763^{* * *}$ & $0.558^{* * *}$ \\
\hline & {$[0.135]$} & {$[0.0824]$} & {$[0.0957]$} \\
\hline \multirow[t]{2}{*}{ Supi } & $0.733^{* * *}$ & $0.958^{* * *}$ & $0.685^{* * *}$ \\
\hline & {$[0.0664]$} & {$[0.0752]$} & {$[0.0912]$} \\
\hline \multirow[t]{2}{*}{ Supc } & $1.011^{* * *}$ & $1.296^{* * *}$ & $1.051^{* * *}$ \\
\hline & {$[0.0727]$} & {$[0.0819]$} & {$[0.0974]$} \\
\hline \multirow[t]{2}{*}{ Montevideo } & $0.342^{* * *}$ & $0.353^{* * *}$ & $0.159^{* * *}$ \\
\hline & {$[0.0255]$} & {$[0.0243]$} & {$[0.0182]$} \\
\hline \multirow[t]{2}{*}{ Constante } & $1.981^{* * *}$ & $1.804^{* * *}$ & $2.622^{* * *}$ \\
\hline & {$[0.195]$} & {$[0.222]$} & {$[0.212]$} \\
\hline \multicolumn{4}{|c|}{ Ecuación de selección } \\
\hline \multirow[t]{2}{*}{ Edad } & $0.243^{* * *}$ & $0.252^{* * *}$ & $0.290^{* * *}$ \\
\hline & {$[0.00967]$} & {$[0.00963]$} & {$[0.00812]$} \\
\hline \multirow[t]{2}{*}{ Edad2 } & $-0.00315^{* * *}$ & $-0.00317^{* * *}$ & $-0.00360^{* * *}$ \\
\hline & {$[0.000128]$} & {$[0.000128]$} & {$[0.000108]$} \\
\hline \multirow[t]{2}{*}{ Pric } & $0.438^{* * *}$ & $0.287^{* * *}$ & $1.121^{* * *}$ \\
\hline & {$[0.0764]$} & {$[0.101]$} & {$[0.101]$} \\
\hline \multirow[t]{2}{*}{ Seci } & $0.705^{* * *}$ & $0.576^{* * *}$ & $1.486^{* * *}$ \\
\hline & {$[0.0747]$} & {$[0.0972]$} & {$[0.0941]$} \\
\hline \multirow[t]{2}{*}{ Secc } & $0.790 * * *$ & $0.879^{* * *}$ & $1.807 * * *$ \\
\hline & {$[0.234]$} & [0.119] & {$[0.102]$} \\
\hline \multirow[t]{2}{*}{ Supi } & $0.898^{* * *}$ & $0.857^{* * *}$ & $1.660^{* * *}$ \\
\hline & {$[0.0940]$} & {$[0.110]$} & [0.0999] \\
\hline \multirow[t]{2}{*}{ Supc } & $1.393^{* * *}$ & $1.127^{* * *}$ & $2.187^{* * *}$ \\
\hline & [0.131] & [0.131] & [0.113] \\
\hline \multirow[t]{2}{*}{ Casado } & $-0.589 * * *$ & $-0.486 * * *$ & $-0.214^{* * *}$ \\
\hline & {$[0.0571]$} & {$[0.0545]$} & {$[0.0475]$} \\
\hline \multirow[t]{2}{*}{ Asiste } & $-1.021^{* * *}$ & $-0.767 * * *$ & $-0.591 * * *$ \\
\hline & {$[0.0551]$} & {$[0.0532]$} & {$[0.0417]$} \\
\hline \multirow[t]{2}{*}{ Estado jefe } & 0.0575 & $0.133^{* * *}$ & $0.134^{* * *}$ \\
\hline & {$[0.0444]$} & {$[0.0411]$} & {$[0.0354]$} \\
\hline \multirow[t]{2}{*}{ ILHH } & $1.08 \mathrm{e}-07$ & $-4.55 \mathrm{e}-07$ & $-7.18 \mathrm{e}-07$ \\
\hline & [8.00e-07] & {$[6.21 \mathrm{e}-07]$} & {$[6.38 \mathrm{e}-07]$} \\
\hline \multirow[t]{2}{*}{ Constante } & $-4.215^{* * *}$ & $-4.535^{* * *}$ & $-6.123^{* * *}$ \\
\hline & [0.181] & {$[0.186]$} & [0.173] \\
\hline Observaciones & 6429 & 6762 & 10798 \\
\hline
\end{tabular}

Fuente: Elaboración propia en base a datos de ECH.

Nota: Errores estándar entre paréntesis. ${ }^{* *}$ significativo al $1 \%,{ }^{* *}$ significativo al $5 \%,{ }^{*}$ significativo al $10 \%$. 
Tabla A5. Estimación de la ecuación de horas trabajadas para jefas de hogar. Método Tobit. Variable dependiente: horas trabajadas.

\begin{tabular}{lccc}
\hline \hline & 1991 & 2000 & 2012 \\
\hline Edad & $1.276^{* * *}$ & $1.225^{* * *}$ & $1.114^{* * *}$ \\
Edad2 & {$[0.368]$} & {$[0.298]$} & {$[0.124]$} \\
& $-0.016^{* * *}$ & $-0.015^{* * *}$ & $-0.014^{* * *}$ \\
Pric & {$[0.004]$} & {$[0.003]$} & {$[0.0014]$} \\
& 1.115 & 2.343 & 0.809 \\
Seci & {$[1.378]$} & {$[1.541]$} & {$[0.993]$} \\
& 1.121 & $4.890^{* * *}$ & $3.605^{* * *}$ \\
Secc & {$[1.375]$} & {$[1.478]$} & {$[0.942]$} \\
& 10.98 & $5.662^{* *}$ & $4.309^{* * *}$ \\
Supi & {$[8.615]$} & {$[2.428]$} & {$[1.024]$} \\
& 1.942 & $4.046^{* *}$ & $3.963^{* * *}$ \\
Supc & {$[2.296]$} & {$[1.945]$} & {$[1.084]$} \\
& -1.026 & $3.983^{* *}$ & $4.416^{* * *}$ \\
Casado & {$[1.737]$} & {$[1.631]$} & {$[0.984]$} \\
& 2.187 & -0.109 & $-0.925^{* *}$ \\
Nro hijos & {$[2.116]$} & {$[1.183]$} & {$[0.456]$} \\
& -0.522 & $-1.011^{* *}$ & $-1.191^{* * *}$ \\
Asiste & {$[0.449]$} & {$[0.395]$} & {$[0.182]$} \\
& $-10.780^{* * *}$ & $-3.716^{*}$ & $-1.852^{* *}$ \\
ILHH & {$[3.096]$} & {$[2.134]$} & {$[0.759]$} \\
& $-0.000^{* * *}$ & 0.000 & -0.000 \\
Constante & {$[4.88 \mathrm{e}-05]$} & {$[2.99 \mathrm{e}-05]$} & {$[1.34 \mathrm{e}-05]$} \\
& $20.470^{* *}$ & $14.980^{* *}$ & $17.060^{* * *}$ \\
\hline Observaciones & {$[8.259]$} & {$[6.321]$} & {$[2.623]$} \\
\hline \hline
\end{tabular}

Fuente: Elaboración propia en base a datos de ECH.

Nota: Errores estándar entre paréntesis. ${ }^{* *}$ significativo al $1 \%,{ }^{* *}$ significativo al $5 \%,{ }^{*}$ significativo al $10 \%$. 
Tabla A6. Estimación de la ecuación de horas trabajadas para cónyuges. Método Tobit. Variable dependiente: horas trabajadas.

\begin{tabular}{|c|c|c|c|}
\hline & 1991 & 2000 & 2012 \\
\hline \multirow[t]{2}{*}{ Edad } & $0.604^{* * *}$ & $0.992^{* * *}$ & $0.898^{* * *}$ \\
\hline & [0.207] & [0.212] & [0.127] \\
\hline \multirow[t]{2}{*}{ Edad2 } & $-0.007^{* * *}$ & $-0.012^{* * *}$ & $-0.012^{* * *}$ \\
\hline & [0.003] & [0.003] & [0.002] \\
\hline \multirow[t]{2}{*}{ Pric } & $2.334^{* * *}$ & $2.977 * *$ & 1.927 \\
\hline & {$[0.878]$} & [1.234] & {$[1.185]$} \\
\hline \multirow[t]{2}{*}{ Seci } & $2.284^{* * *}$ & $5.439 * * *$ & $5.159 * * *$ \\
\hline & {$[0.867]$} & [1.192] & [1.144] \\
\hline \multirow[t]{2}{*}{ Secc } & 3.253 & $4.651^{* * *}$ & $5.757^{* * *}$ \\
\hline & [3.392] & [1.594] & [1.201] \\
\hline \multirow[t]{2}{*}{ Supi } & -1.269 & $5.364^{* * *}$ & $4.770 * * *$ \\
\hline & [1.386] & [1.559] & [1.283] \\
\hline \multirow[t]{2}{*}{ Supc } & $-3.271 * * *$ & $3.517^{* * *}$ & $5.190 * * *$ \\
\hline & [1.021] & [1.304] & [1.184] \\
\hline \multirow[t]{2}{*}{ Nro hijos } & $-1.080 * * *$ & $-1.513^{* * *}$ & $-1.684^{* * *}$ \\
\hline & [0.237] & {$[0.245]$} & [0.178] \\
\hline \multirow[t]{2}{*}{ Asiste } & -1.205 & $-4.914^{* * *}$ & $-1.925^{* *}$ \\
\hline & [1.778] & [1.744] & {$[0.827]$} \\
\hline \multirow[t]{2}{*}{ Estado jefe } & -0.979 & -0.611 & -0.229 \\
\hline & [0.939] & {$[0.915]$} & {$[0.664]$} \\
\hline \multirow[t]{2}{*}{ ILHH } & $1.48 \mathrm{e}-06$ & $-1.06 \mathrm{e}-05$ & $1.68 \mathrm{e}-06$ \\
\hline & {$[6.26 \mathrm{e}-06]$} & {$[9.65 \mathrm{e}-06]$} & {$[7.85 \mathrm{e}-06]$} \\
\hline \multirow[t]{2}{*}{ Constante } & $28.060 * * *$ & $17.360 * * *$ & $18.230^{* * *}$ \\
\hline & [4.066] & [4.198] & {$[2.685]$} \\
\hline Observaciones & 4436 & 4319 & 8589 \\
\hline
\end{tabular}

Nota: Errores estándar entre paréntesis. ${ }^{* *}$ significativo al $1 \%,{ }^{* *}$ significativo al $5 \%,{ }^{*}$ significativo al $10 \%$. 
Tabla A7. Estimación de la ecuación de horas trabajadas para otras mujeres del hogar. Método Tobit. Variable dependiente: horas trabajadas.

\begin{tabular}{lccc}
\hline \hline & 1991 & 2000 & 2012 \\
\hline Edad & $0.562^{* * *}$ & $0.964^{* * *}$ & $1.164^{* * *}$ \\
Edad2 & {$[0.174]$} & {$[0.175]$} & {$[0.124]$} \\
& $-0.007^{* * *}$ & $-0.012^{* * *}$ & $-0.015^{* * *}$ \\
Pric & {$[0.002]$} & {$[0.002]$} & {$[0.002]$} \\
& 0.491 & -0.594 & $3.709^{*}$ \\
Seci & {$[1.453]$} & {$[1.929]$} & {$[2.054]$} \\
& -0.779 & 1.802 & $6.760^{* * *}$ \\
Secc & {$[1.415]$} & {$[1.861]$} & {$[1.965]$} \\
& -2.370 & 2.119 & $7.774^{* * *}$ \\
Supi & {$[3.464]$} & {$[2.079]$} & {$[2.020]$} \\
& $-3.914^{* *}$ & -1.461 & $5.923^{* * *}$ \\
Supc & {$[1.776]$} & {$[2.082]$} & {$[2.043]$} \\
& $-8.789^{* * *}$ & -2.516 & $5.250^{* * *}$ \\
Casado & {$[1.697]$} & {$[2.050]$} & {$[2.029]$} \\
& $-4.368^{* * *}$ & $-2.337^{* *}$ & -0.655 \\
Asiste & {$[0.939]$} & {$[0.912]$} & {$[0.652]$} \\
& $-8.690^{* * *}$ & $-6.533^{* * *}$ & $-5.692^{* * *}$ \\
Estado jefe & {$[1.066]$} & {$[1.004]$} & {$[0.632]$} \\
& -0.303 & $-1.345^{* *}$ & 0.671 \\
ILHH & {$[0.731]$} & {$[0.675]$} & {$[0.488]$} \\
& $5.84 \mathrm{e}-05^{* * *}$ & $3.53 \mathrm{e}-05^{* * *}$ & $2.60 \mathrm{e}-06$ \\
Constante & {$[1.35 \mathrm{e}-05]$} & {$[9.77 \mathrm{e}-06]$} & {$[8.68 \mathrm{e}-06]$} \\
& $32.860^{* * *}$ & $22.660^{* * * *}$ & $10.850^{* * *}$ \\
\hline Observaciones & {$[3.276]$} & {$[3.405]$} & {$[2.924]$} \\
\hline \hline
\end{tabular}

Fuente: Elaboración propia en base a datos de ECH.

Nota: Errores estándar entre paréntesis. ${ }^{* *}$ significativo al $1 \%,{ }^{* *}$ significativo al $5 \%,{ }^{*}$ significativo al $10 \%$. 
Tabla A8. Descomposición del cambio en la brecha de pobreza: Uruguay 1991-2012.

\begin{tabular}{lccc}
\hline \hline & \multicolumn{3}{c}{ Valor del indicador } \\
& 1991 & 2000 & 2012 \\
\hline FGT(1) & 3.32 & 3.30 & 2.40 \\
\hline & \multicolumn{3}{c}{ Descomposición } \\
& $1991-2000$ & $2000-2012$ & $1991-2012$ \\
\hline Variación observada & -0.02 & -0.90 & -0.92 \\
Efectos & & & \\
\hline Empleo & -0.19 & -0.23 & $-0.42^{* *}$ \\
Estado & -0.04 & -0.12 & -0.20 \\
Horas trabajadas & -0.14 & -0.20 & $-0.31^{*}$ \\
\hline Retornos a la educación & 0.03 & -0.15 & -0.10 \\
Retornos a la experiencia & 0.16 & -0.38 & -0.31 \\
Inobservables & 0.01 & -0.07 & -0.06 \\
\hline \hline
\end{tabular}

Fuente: Elaboración propia en base a ECH.

Nota: $* * *$ significativo al $1 \%,{ }^{* *}$ significativo al $5 \%,{ }^{*}$ significativo al $10 \%$. Localidades urbanas con más de 5000 hab.

Tabla A9. Descomposición del cambio en la profundidad de la pobreza: Uruguay 1991-2012.

\begin{tabular}{lccc}
\hline \hline & \multicolumn{3}{c}{ Valor del indicador } \\
& 1991 & 2000 & 2012 \\
\hline FGT(2) & 1.53 & 1.47 & 1.04 \\
\hline & \multicolumn{3}{c}{ Descomposición } \\
& $1991-2000$ & $2000-2012$ & $1991-2012$ \\
\hline Variación observada & -0.06 & -0.43 & -0.49 \\
Efectos & & & \\
\hline Empleo & -0.04 & -0.20 & -0.24 \\
Estado & -0.04 & -0.14 & -0.13 \\
Horas trabajadas & -0.01 & -0.18 & -0.18 \\
\hline Retornos a la educación & 0.09 & -0.16 & -0.06 \\
Retornos a la experiencia & 0.15 & -0.30 & -0.17 \\
Inobservables & 0.07 & -0.12 & -0.04 \\
\hline \hline
\end{tabular}

Fuente: Elaboración propia en base a ECH.

Nota: ${ }^{* *}$ significativo al $1 \%,{ }^{* *}$ significativo al $5 \%,{ }^{*}$ significativo al $10 \%$. Localidades urbanas con más de 5000 hab. 